\title{
Localization of two-dimensional massless Dirac fermions in a magnetic quantum dot
}

\author{
Martin Könenberg and Edgardo Stockmeyer ${ }^{1}$
}

\begin{abstract}
We consider a two-dimensional massless Dirac operator $H$ in the presence of a perturbed homogeneous magnetic field $B=B_{0}+b$ and a scalar electric potential $V$. For $V \in L_{\mathrm{loc}}^{p}\left(\mathbb{R}^{2}\right), p \in(2, \infty]$, and $b \in L_{\mathrm{loc}}^{q}\left(\mathbb{R}^{2}\right), q \in(1, \infty]$, both decaying at infinity, we show that states in the discrete spectrum of $H$ are superexponentially localized. We establish the existence of such states between the zeroth and the first Landau level assuming that $V=0$. In addition, under the condition that $b$ is rotationally symmetric and that $V$ satisfies certain analyticity condition on the angular variable, we show that states belonging to the discrete spectrum of $H$ are Gaussian-like localized.
\end{abstract}

Mathematics Subject Classification (2010). Primary 81Q10; Secondary 47B25.

Keywords. Magnetic operator, localization, Dirac operator.

\section{Introduction}

Graphene is a two-dimensional lattice of carbon atoms arranged on a honeycomb structure. Due to its unusual properties it has attracted a great deal of attention since its discovery; see [4] and [21]. One of the striking facts about graphene is that the dynamics of its low-energy excitations (the charge carriers) can be described by massless two-dimensional Dirac operators. An interesting feature of such Dirac fermions is the lack of localization under the influence of an external electric potential; see [31] and [15]. This fact, related to Klein's paradox [4], is due to the peculiar conelike gapless structure of the spectrum of massless free Dirac operators.

It was suggested in [7] that it is possible to confine such massless Dirac fermions in graphene by inhomogeneous magnetic fields of the type $B=B_{0}+b$, where $B_{0}>0$ is a constant and $b$ a perturbation with negative flux that decays at infinity. The spectrum of the corresponding Dirac operator in a constant magnetic field $B_{0}$ is given by the (relativistic) Landau levels. The idea is that as the perturbation $b$ is turned on eigenvalues will emerge from the Landau levels giving rise to states localized on the bulk of the support of $b$. In this manner a so-called (magnetic) quantum dot or

\footnotetext{
${ }^{1}$ Both authors have been partially supported by the DFG (SFB/TR12).
} 
artificial atom can be created. These type of models, also with an external electric potential $V$, have been further studied in the physics literature, for instance in [8], [22], [32], and [16] for the one particle case and in [13] and [9] for the multiparticle case. The articles [8], [22], [32], and [16] deal with specific electromagnetic fields for which the model is partly solvable or suitable for numerical computations.

In this article we consider a large class of electromagnetic perturbations $(b, V)$ with $V \in L_{\mathrm{loc}}^{p}\left(\mathbb{R}^{2}\right), p \in(2, \infty]$, and $b \in L_{\mathrm{loc}}^{q}\left(\mathbb{R}^{2}\right), q \in(1, \infty]$, both decaying at infinity. The essential spectrum of the corresponding massless Dirac-operator $H$ describing the quantum dot is given by the Landau levels. We show that eigenfunctions belonging to the discrete spectrum of $H$ are superexponentially localized, i.e., they decay faster than any exponential. In the case when $V=0$ we verify the existence of eigenvalues between the zeroth and the first Landau-level assuming that $b<0$. Assuming that a certain analyticity conditions on the angular variable of $V$ is fulfilled and that $b$ is rotationally symmetric we prove that those states are actually Gaussian-like localized. These type of results on superexponential and Gaussian localization, although new for Dirac operators, are known to hold for spinless magnetic Schrödinger operators [6], [10], [19], and [29]. We benefit from this insight to prove our statements. A precise description of our results is given in the next section.

Acknowledgements. Edgardo Stockmeyer thanks Horia Cornean for stimulating discussions at the conference Spectral days in Santiago de Chile.

\section{Results}

We consider the massless two-dimensional Dirac operator with an external magnetic field $B: \mathbb{R}^{2} \rightarrow \mathbb{R}$, pointing perpendicularly to the plane, and an electric potential $V: \mathbb{R}^{2} \rightarrow \mathbb{R}$. We are interested in the Hamiltonians

$$
\begin{aligned}
D_{\mathbf{A}} & \stackrel{\text { def }}{=} \sigma \cdot(\mathbf{p}-\mathbf{A}), \\
H & \stackrel{\text { def }}{=} D_{\mathbf{A}}+V,
\end{aligned}
$$

a priori defined on $C_{0}^{\infty}\left(\mathbb{R}^{2} ; \mathbb{C}^{2}\right) \subset L^{2}\left(\mathbb{R}^{2} ; \mathbb{C}^{2}\right)$. Here $\mathbf{p} \stackrel{\text { def }}{=} \frac{1}{\mathrm{i}} \nabla$ is the momentum of the particle and $\sigma \stackrel{\text { def }}{=}\left(\sigma_{1}, \sigma_{2}\right)$ is a vector whose entries

$$
\sigma_{1} \stackrel{\text { def }}{=}\left(\begin{array}{ll}
0 & 1 \\
1 & 0
\end{array}\right), \quad \sigma_{2} \stackrel{\text { def }}{=}\left(\begin{array}{cc}
0 & -\mathrm{i} \\
\mathrm{i} & 0
\end{array}\right),
$$

are Pauli matrices. The magnetic field $B$ enters in the definitions (1) and (2) by means of the magnetic vector potential $\mathbf{A}=\left(A_{1}, A_{2}\right): \mathbb{R}^{2} \rightarrow \mathbb{R}^{2}$ through the relation

$$
B=\partial_{1} A_{2}-\partial_{2} A_{1} \stackrel{\text { def }}{=} \operatorname{curl} \mathbf{A},
$$

which is understood in the sense of distributions. 
Throughout this article we assume the following on $(B, V)$.

(A1) $B=B_{0}+b$ where $B_{0}>0$ is a number and $b \in L_{\text {loc }}^{q}\left(\mathbb{R}^{2} ; \mathbb{R}\right)$ for some $q \in(1, \infty]$ and $\lim _{n \rightarrow \infty}\left\|1_{\{|\mathbf{x}| \geq n\}} b\right\|_{\infty}=0$.

(A2) $V \in L_{\mathrm{loc}}^{p}\left(\mathbb{R}^{2} ; \mathbb{R}\right)$ for some $p \in(2, \infty]$ and $\lim _{n \rightarrow \infty}\left\|\mathbb{1}_{\{|\mathbf{x}| \geq n\}} V\right\|_{\infty}=0$.

Here $\mathbb{1}_{I}(\cdot)$ denotes the characteristic function on the set $I$. Assuming that $B$ fulfills (A1) we can always find $\mathbf{A} \in L_{\text {loc }}^{t}\left(\mathbb{R}^{2} ; \mathbb{R}^{2}\right)$ for some $t \in(2, \infty]$ satisfying (3); see Remark 8 . For such magnetic vector potentials and electric potentials $V$ satisfying (A2) we know that the operators defined in (1) and (2) are essentially self-adjoint; see Subsection 3.1. We denote their self-adjoint extensions by the same symbols and their domains by $\mathscr{D}\left(D_{\mathbf{A}}\right)$ and $\mathscr{D}(H)$ respectively.

To the homogeneous magnetic field $B_{0}$ we associate the vector potential

$$
\mathbf{A}_{0} \stackrel{\text { def }}{=} \frac{B_{0}}{2}\left(-x_{2}, x_{1}\right)
$$

satisfying curl $\mathbf{A}_{0}=B_{0}$. It is well known that the spectrum of $D_{\mathbf{A}_{0}}$ consists of infinitely degenerated eigenvalues $\left(l_{n}\right)_{n \in \mathbb{Z}}$, called Landau levels, given by

$$
l_{n} \stackrel{\text { def }}{=} \operatorname{sgn}(n) \sqrt{2|n| B_{0}}, \quad n \in \mathbb{Z},
$$

where $\operatorname{sgn}(n)=n /|n|$ if $n \neq 0$ and equals one if $n=0$.

Given a self-adjoint operator $T$ we write $\sigma_{\mathrm{pp}}(T), \sigma_{\mathrm{d}}(T)$, and $\sigma_{\text {ess }}(T)$ to denote the pure point, discrete, and essential spectra of $T$ respectively. Our first main result is as follows.

Theorem 1. Assume that $B$ satisfies (A1) and let $\mathbf{A} \in L_{\mathrm{loc}}^{p}\left(\mathbb{R}^{2} ; \mathbb{R}^{2}\right), p \in(2, \infty]$, with curl $\mathbf{A}=B$. Then, the spectrum of $D_{\mathbf{A}}$ is symmetric with respect to zero and

$$
\sigma_{\mathrm{ess}}\left(D_{\mathbf{A}}\right)=\left(l_{n}\right)_{n \in \mathbb{Z}}
$$

\section{Moreover,}

(a) if $b \leq 0$ and strictly negative on some open set, then the discrete spectrum of $D_{\mathrm{A}}$ on $\left(0, l_{1}\right)$ is non-empty, i.e., $\sigma_{\mathrm{d}}\left(D_{\mathbf{A}}\right) \cap\left(0, l_{1}\right) \neq \varnothing$ and

$$
\operatorname{dim}\left(\operatorname{Ran}\left(\mathbb{1}_{\left(0, l_{1}\right)}\left(D_{\mathrm{A}}\right)\right)\right)=\infty ;
$$

(b) if $b \geq 0$ then

$$
\operatorname{dim}\left(\operatorname{Ran}\left(\mathbb{1}_{\left(0, l_{1}\right)}\left(D_{\mathbf{A}}\right)\right)\right)=0
$$

This theorem is a consequence of Lemmata 2 and 3. That the spectrum of $D_{\mathbf{A}}$ is symmetric with respect to zero is well known; see, however, Proposition 1. 
Remark 1. A similar result to Theorem 1 is shown in [3] when $b$ is replaced by $\lambda b$ and $\lambda$ is assumed to be sufficiently large. Moreover, in [3] stronger regularity assumptions on $b$ are made. In addition, the magnetic vector potential a associated to $b$ is assumed to decay at infinity. However, the results of [3] hold for more general background magnetic fields than $B_{0}$. We note also that our proof differs from the one in [3].

Remark 2. For Schrödinger and Pauli operators the spectral subspaces obtained by splitting the Landau levels with electromagnetic perturbations decaying at infinity have been investigated in the last years; see e.g. [25] and references therein.

Remark 3. Assume that (A1) and (A2) are fulfilled. As a consequence of Lemmata 1 and 2 below,

$$
\sigma_{\text {ess }}(H)=\sigma_{\text {ess }}\left(D_{\mathbf{A}}\right)=\sigma_{\text {ess }}\left(D_{\mathbf{A}_{0}}\right),
$$

for any $\mathbf{A} \in L_{\mathrm{loc}}^{p}\left(\mathbb{R}^{2} ; \mathbb{R}^{2}\right), p \in(2, \infty]$, with $\operatorname{curl} \mathbf{A}=B$.

Our next result state that eigenfunctions corresponding to the discrete spectrum of $H$ are super-exponentially localized.

Theorem 2. Assume that $B$ and $V$ satisfy (A1) and (A2) respectively and let $\mathbf{A} \in$ $L_{\text {loc }}^{p}\left(\mathbb{R}^{2} ; \mathbb{R}^{2}\right), p \in(2, \infty]$, with curl $\mathbf{A}=B=B_{0}+b$. Then, for any eigenfunction $\Psi$ of $H=D_{\mathbf{A}}+V$ with $H \Psi=E \Psi$ and $E \in \mathbb{R} \backslash \sigma\left(D_{\mathbf{A}_{0}}\right)$ the following holds: for every $r \in[2, \infty]$ and $\gamma>0$ there exists an $R>0$ such that

$$
\left\|1_{\{|\mathbf{x}| \geq R\}} e^{\gamma|\mathbf{x}|} \Psi\right\|_{r}<\infty .
$$

This theorem is proven in Section 5.

Remark 4. This type of results are known to hold for magnetic Schrödinger operators $(\mathbf{p}-\mathbf{A})^{2}+B$. Our proof follows the ideas presented in [6]. In fact, since our operator is linear in $\mathbf{A}$, some parts of the argument are more straightforward. For instance, we do not require that $b \in C^{1}\left(\mathbb{R}^{2} ; \mathbb{R}\right)$ decays in the $C^{1}$-norm as done in [6].

Remark 5. One essential ingredient in the proof of Theorem 2 is the explicit knowledge of the Green function $G_{0}$ of $D_{\mathbf{A}_{0}}$. This is calculated in Appendix A.

In order to obtain Gaussian decay we make further assumptions on $(B, V)$. Let $T=\mathbb{R} /(2 \pi \mathbb{Z})$ and let $v=v(r, \theta),(r, \theta) \in \mathbb{R}^{+} \times T$ be the potential $V$ written in polar coordinates. We assume the following.

(A3) $B$ is radially symmetric, i.e., $b(\mathbf{x})=b(r), r=|\mathbf{x}|$. 
(A4) For any $(r, \theta) \in \mathbb{R}^{+} \times T$ the mapping $\mathbb{R} \ni a \mapsto v(r, \theta+a) \stackrel{\text { def }}{=} v_{a}(r, \theta)$ has an analytic continuation $\tilde{v}_{z}(r, \theta)$ to $\mathbb{C}$. Moreover, for any $\tau>0$ there exist a $p \in(2, \infty]$ and a real-valued function $u_{\tau} \in L_{\mathrm{loc}}^{p}\left(\mathbb{R}^{+} \times T, r d r d \theta\right)$ such that $\left\|\mathbb{1}_{\{r>n\}} u_{\tau}\right\|_{\infty} \rightarrow 0$ as $n \rightarrow \infty$ and

$$
\left|\tilde{v}_{z}(r, \theta)\right| \leq u_{\tau}(r, \theta)
$$

for any $(r, \theta) \in \mathbb{R}^{+} \times T$ and $z \in S_{\tau} \stackrel{\text { def }}{=}\{z \in \mathbb{C}:|\operatorname{Im} z| \leq \tau\}$.

(A5) $v$ is differentiable with respect to $r$ and $\mathbb{R} \ni a \mapsto \partial_{r} v(r, \theta+a)$ can be analytically continued to $\partial_{r} \tilde{v}_{z}(r, \theta)$ on $\mathbb{C}$. Moreover, there exist a $\rho>0$ such that for any $\tau>0$ there is $\kappa_{\tau}>0$ such that $\left|\mathbb{1}_{\{r>\rho\}} \partial_{r} v_{z}(r, \theta)\right| \leq \kappa_{\tau}$ for any $(r, \theta) \in \mathbb{R}^{+} \times T$ and $z \in S_{\tau}$.

Theorem 3. Assume that $B$ satisfies (A1) and (A3) and $V$ satisfies (A2), (A4), and (A5). Let $\mathbf{A} \in L_{\text {loc }}^{p}\left(\mathbb{R}^{2} ; \mathbb{R}^{2}\right), p \in(2, \infty]$, with curl $\mathbf{A}=B$. Then, for any eigenfunction $\Psi$ of $H=D_{\mathbf{A}}+V$ with $H \Psi=E \Psi$ and $E \in \mathbb{R} \backslash \sigma\left(D_{\mathbf{A}_{0}}\right)$ the following holds: For every $0<\alpha<1$, we have

$$
\left\|e^{\alpha B_{0} / 4|\mathbf{x}|^{2}} \Psi\right\|_{2}<\infty .
$$

This theorem is proven in Section 6.

Remark 6. The analyticity assumption (A4) on the angular variable of $V$ implies, by a Paley-Wiener argument, exponential decay of the Fourier modes of the potential in its angular momentum decomposition; see (40), (41), and (50) below. Assumption (A5) is similar to (A4) but for the radial derivative of the potential.

Remark 7. The first proof of Gaussian localization for magnetic Schrödinger operators using assumptions like (A4), but not (A5), was given in [10]. In addition, an example of a potential decaying at infinity for which the corresponding ground state decays slower than a Gaussian is also given in [10]. The proof in [10] is based on a generalized Feynman-Kac formula. An alternative proof using Agmon-type estimates with localizations in space and angular momentum was given in [19]. A variation of the method in [19] was used in [29] to treat the general $n$-dimensional case, again for magnetic Schrödinger operators. Our proof follows the ideas developed in [19]. However, it turns out to be more involved since our operator is not bounded from below. To overcome this difficulty we square the Dirac operator (or parts of it). This is the reason why (A5) is used in our setting.

The article is organized as follows. In Section 3 we review some essentially well known facts about magnetic Dirac operators. Sections 4, 5, and 6 are devoted to the proofs of theorems 1,2 , and 3 respectively. The article ends with an appendix containing some useful technical results. 


\section{Preliminaries}

3.1. Essential self-adjointness. Throughout this article we consider magnetic potentials $\mathbf{A} \in L_{\mathrm{loc}}^{p}\left(\mathbb{R}^{2} ; \mathbb{R}^{2}\right)$ and electric potentials $V \in L_{\mathrm{loc}}^{q}\left(\mathbb{R}^{2}\right), p, q \in(2, \infty]$. In order to show essential self-adjointness of the operators $H$ and $D_{\mathrm{A}}$ defined in (1) and (2) it suffices to prove that

$$
H_{R} \varphi \stackrel{\text { def }}{=} \sigma \cdot\left(\mathbf{p}-\mathbb{1}_{\{|\mathbf{x}| \leq R\}} \mathbf{A}\right) \varphi+\mathbb{1}_{\{|\mathbf{x}| \leq R\}} V \varphi, \quad \varphi \in C_{0}^{\infty}\left(\mathbb{R}^{2} ; \mathbb{C}^{2}\right),
$$

is essentially self-adjoint for every $R>0$; see [5]. Using that for $f \in L^{p}\left(\mathbb{R}^{2} ; \mathbb{C}\right)$ and $2<p<\infty$

$$
f(\mathbf{x})\left(\mathbf{p}^{2}+1\right)^{-1 / 2}
$$

is a compact operator (see [28], Theorem 4.1) we get that $\mathbb{1}_{\{|\mathbf{x}| \leq R\}}(V-\sigma \cdot \mathbf{A}$ ) is a relative compact perturbation of $D_{0}$. This shows essential self-adjointness of $H_{R}$, since $D_{0}$ is essentially self-adjoint on $C_{0}^{\infty}\left(\mathbb{R}^{2} ; \mathbb{C}^{2}\right)$.

3.2. Gauge invariance. Let $\mathbf{A}, \widehat{\mathbf{A}} \in L_{\mathrm{loc}}^{p}\left(\mathbb{R}^{2} ; \mathbb{R}^{2}\right), 2<p<\infty$, be two vector potentials with

$$
\operatorname{curl} \mathbf{A}=\operatorname{curl} \widehat{\mathbf{A}}
$$

in the sense of distributions. According to [17] there is a gauge function $\widehat{\Phi} \in$ $W_{\text {loc }}^{1, p}\left(\mathbb{R}^{2} ; \mathbb{R}\right)$ such that

$$
\mathbf{A}=\widehat{\mathbf{A}}+\nabla \widehat{\Phi} .
$$

It follows, for any electric potential $V \in L_{\mathrm{loc}}^{q}\left(\mathbb{R}^{2} ; \mathbb{R}\right), q \in(2, \infty]$, that

$$
\left(D_{\mathbf{A}}+V\right)=e^{\mathrm{i} \hat{\Phi}}\left(D_{\widehat{\mathbf{A}}}+V\right) e^{-\mathrm{i} \hat{\Phi}} .
$$

In particular, $e^{\mathrm{i} \hat{\Phi}}\left(D_{\widehat{\mathbf{A}}}+V\right) e^{-\mathrm{i} \hat{\Phi}}$ is essentially self-adjoint on $C_{0}^{\infty}\left(\mathbb{R}^{2} ; \mathbb{C}^{2}\right)$.

This can be seen as follows. Note that

$$
\mathscr{D}\left(e^{\mathrm{i} \widehat{\Phi}}\left(D_{\widehat{\mathbf{A}}}+V\right) e^{-\mathrm{i} \hat{\Phi}}\right)=\left\{f \in L^{2}\left(\mathbb{R}^{2} ; \mathbb{C}^{2}\right): e^{-\mathrm{i} \widehat{\Phi}} f \in \mathscr{D}\left(D_{\widehat{\mathbf{A}}}+V\right)\right\} .
$$

Pick functions $\eta, \eta^{\prime} \in C_{0}^{\infty}\left(\mathbb{R}^{2} ; \mathbb{C}^{2}\right)$ and a sequence $\left(\widehat{\Phi}_{m}\right)_{m \in \mathbb{N}}$ in $C^{\infty}\left(\mathbb{R}^{2} ; \mathbb{R}\right)$ with $\widehat{\Phi}_{m} \rightarrow \widehat{\Phi}$ in $W_{\mathrm{loc}}^{1, p}\left(\mathbb{R}^{2}\right)$ (and hence in $W_{\mathrm{loc}}^{1,2}\left(\mathbb{R}^{2}\right)$ ) as $m \rightarrow \infty$. Then,

$$
\begin{aligned}
& \left\langle\left(D_{\widehat{\mathbf{A}}}+V\right) \eta^{\prime}, e^{-\mathrm{i} \widehat{\Phi}} \eta\right\rangle=\lim _{m \rightarrow \infty}\left\langle\left(D_{\widehat{\mathbf{A}}}+V\right) \eta^{\prime}, e^{-\mathrm{i} \widehat{\Phi}_{m}} \eta\right\rangle \\
& =\lim _{m \rightarrow \infty}\left\langle e^{\mathrm{i} \widehat{\Phi}_{m}} \eta^{\prime},\left(D_{\widehat{\mathbf{A}}}+V\right) \eta\right\rangle-\lim _{m \rightarrow \infty}\left\langle e^{\mathrm{i} \widehat{\Phi}_{m}} \eta^{\prime}, \boldsymbol{\sigma} \cdot \nabla \widehat{\Phi}_{m} \eta\right\rangle \\
& =\left\langle e^{\mathrm{i} \hat{\Phi}} \eta^{\prime},\left(D_{\widehat{\mathbf{A}}}+V\right) \eta\right\rangle-\left\langle e^{\mathrm{i} \widehat{\Phi}} \eta^{\prime}, \sigma \cdot \nabla \widehat{\Phi} \eta\right\rangle .
\end{aligned}
$$

Since $\eta^{\prime}$ is an arbitrary element of a core of $D_{\widehat{\mathbf{A}}}+V$, it follows that

$$
e^{-\mathrm{i} \hat{\Phi}} \eta \in \mathscr{D}\left(D_{\widehat{\mathbf{A}}}+V\right)
$$


and

$$
\left(D_{\widehat{\mathbf{A}}}+V\right) e^{-\mathrm{i} \widehat{\Phi}} \eta=e^{-\mathrm{i} \widehat{\Phi}}\left(D_{\widehat{\mathbf{A}}}+V-\sigma \cdot \nabla \widehat{\Phi}\right) \eta,
$$

which implies that

$$
e^{\mathrm{i} \widehat{\Phi}}\left(D_{\widehat{\mathbf{A}}}+V\right) e^{-\mathrm{i} \widehat{\Phi}} \eta=\left(D_{\mathbf{A}}+V\right) \eta, \quad \eta \in C_{0}^{\infty}\left(\mathbb{R}^{2} ; \mathbb{C}^{2}\right) .
$$

Due to the essential self-adjointness of $D_{\mathbf{A}}+V$ we deduce that $e^{i \widehat{\Phi}}\left(D_{\widehat{\mathbf{A}}}+V\right) e^{-\mathrm{i} \hat{\Phi}}$ is also essentially self-adjoint on $C_{0}^{\infty}\left(\mathbb{R}^{2} ; \mathbb{C}^{2}\right)$ and that the two operators coincide.

3.3. Supersymmetry. For $\mathbf{A}=\left(A_{1}, A_{2}\right)$ with $A_{j} \in L_{\mathrm{loc}}^{p}\left(\mathbb{R}^{2}\right), p \in(2, \infty], j=$ 1,2 , we define the following two operators

$$
\begin{array}{ll}
d_{1} \varphi=\left[\left(p_{1}-A_{1}\right)+\mathrm{i}\left(p_{2}-A_{2}\right)\right] \varphi, & \varphi \in C_{0}^{\infty}\left(\mathbb{R}^{2} ; \mathbb{C}\right), \\
d_{2} \varphi=\left[\left(p_{1}-A_{1}\right)-\mathrm{i}\left(p_{2}-A_{2}\right)\right] \varphi, & \varphi \in C_{0}^{\infty}\left(\mathbb{R}^{2} ; \mathbb{C}\right) .
\end{array}
$$

Clearly, we have that

$$
D_{\mathbf{A}}\left\lceil C_{0}^{\infty}\left(\mathbb{R}^{2} ; \mathbb{C}^{2}\right)=\left(\begin{array}{cc}
0 & d_{2} \\
d_{1} & 0
\end{array}\right) .\right.
$$

Since $D_{\mathbf{A}}\left\lceil C_{0}^{\infty}\left(\mathbb{R}^{2} ; \mathbb{C}^{2}\right)\right.$ is essentially self-adjoint it follows that $d_{1}$ and $d_{2}$ are closable; see [30], Section 5.2.2. In addition, setting $d \stackrel{\text { def }}{=} \overline{d_{1}}$ one finds that $d^{*}=\overline{d_{2}}$ and

$$
D_{\mathbf{A}}=\left(\begin{array}{cc}
0 & d^{*} \\
d & 0
\end{array}\right) \quad \text { on } \mathscr{D}\left(D_{\mathbf{A}}\right)=\mathscr{D}(d) \oplus \mathscr{D}\left(d^{*}\right) .
$$

It is known that $d d^{*}$ and $d^{*} d$ are self-adjoint with domains $\mathscr{D}\left(d d^{*}\right)=\{\varphi \in$ $\left.\mathscr{D}\left(d^{*}\right): d^{*} \varphi \in \mathscr{D}(d)\right\}$ and $\mathscr{D}\left(d^{*} d\right)=\left\{\varphi \in \mathscr{D}(d): d \varphi \in \mathscr{D}\left(d^{*}\right)\right\}$. Moreover, there is a unitary map $S$ from $\operatorname{Ker}\left(\mathrm{dd}^{*}\right)^{\perp}$ to $\operatorname{Ker}\left(\mathrm{d}^{*} \mathrm{~d}\right)^{\perp}$, such that

$$
d d^{*} \uparrow_{\operatorname{Ker}\left(\mathrm{dd}^{*}\right)^{\perp}}=S^{*} d^{*} d \uparrow_{\operatorname{Ker}\left(\mathrm{d}^{*} \mathrm{~d}\right) \perp}^{\perp} .
$$

Let us note that we can block-diagonalize $D_{\mathrm{A}}$ using the Foldy-Wouthuysen transformation. Setting

$$
\begin{aligned}
& a_{+}= \begin{cases}1 / \sqrt{2} & \text { on } \operatorname{Ker}\left(D_{\mathbf{A}}\right)^{\perp}, \\
1 & \text { on } \operatorname{Ker}\left(D_{\mathbf{A}}\right),\end{cases} \\
& a_{-}= \begin{cases}1 / \sqrt{2} & \text { on } \operatorname{Ker}\left(D_{\mathbf{A}}\right)^{\perp}, \\
0 & \text { on } \operatorname{Ker}\left(D_{\mathbf{A}}\right),\end{cases}
\end{aligned}
$$

we define the Foldy-Wouthuysen transformation as

$$
U=a_{+}+\sigma_{3} \operatorname{sgn}\left(D_{\mathbf{A}}\right) a_{-},
$$


where $\operatorname{sgn}\left(D_{\mathbf{A}}\right)=D_{\mathbf{A}} /\left|D_{\mathbf{A}}\right|$ on $\operatorname{Ker}\left(D_{\mathbf{A}}\right)^{\perp}$ and equals zero on $\operatorname{Ker}\left(D_{\mathbf{A}}\right)$ and

$$
\sigma_{3}=\left(\begin{array}{cc}
1 & 0 \\
0 & -1
\end{array}\right)
$$

The unitarity of the above transformation can be easily verified observing that $\varphi \in$ $\operatorname{Ker}\left(D_{\mathbf{A}}\right) \Leftrightarrow \sigma_{3} \varphi \in \operatorname{Ker}\left(D_{\mathbf{A}}\right)$ and that $\sigma_{3} \operatorname{sgn}\left(D_{\mathbf{A}}\right)=-\operatorname{sgn}\left(D_{\mathbf{A}}\right) \sigma_{3}$. The latter relation holds since $\sigma_{3} D_{\mathbf{A}}=-D_{\mathbf{A}} \sigma_{3}$ and $\sigma_{3}\left|D_{\mathbf{A}}\right|=\left|D_{\mathbf{A}}\right| \sigma_{3}$. A direct computation yields

$$
U D_{\mathrm{A}} U^{*}=\left(\begin{array}{cc}
\sqrt{d^{*} d} & 0 \\
0 & -\sqrt{d d^{*}}
\end{array}\right) .
$$

Equation (7) and (8) imply the following statement.

Proposition 1. Let $\mathbf{A} \in L_{\mathrm{loc}}^{p}\left(\mathbb{R}^{2} ; \mathbb{R}^{2}\right)$ for some $p \in(2, \infty]$. Then, the spectrum of $D_{\mathbf{A}}$ is symmetric with respect to zero and

$$
\sigma_{\sharp}\left(D_{\mathbf{A}}\right) \cap(0, \infty)=\sigma_{\sharp}\left(\sqrt{d^{*} d}\right) \backslash\{0\}, \quad \sharp \in\{\text { pp, d, ess }\} .
$$

\section{The spectrum of $D_{\mathrm{A}}$}

The aim of this section is to show Theorem 1. An important ingredient is the study of the essential spectrum of $D_{\mathrm{A}}$. In order to do that we modify an argument from [14] obtaining Lemma 1 below. We combine this with a result from [24] on the infiniteness of zero modes for Pauli operators (see Lemma 2 below). The proof of the theorem is then a consequence of Lemmata 2 and 3.

In the following discussion we assume that $B=B_{0}+b$ with $B_{0}>0$ and $b \in$ $L_{\text {loc }}^{1}\left(\mathbb{R}^{2} ; \mathbb{R}\right)$ such that $|b|^{1 / 2}$ is relative $\sqrt{\mathbf{p}^{2}+1}$-compact. Let $\mathbf{A} \in L_{\text {loc }}^{p}\left(\mathbb{R}^{2} ; \mathbb{R}^{2}\right), p \in$ $(2, \infty]$, with curl $\mathbf{A}=B$. We start by observing that, for $\varphi \in C_{0}^{\infty}\left(\mathbb{R}^{2} ; \mathbb{C}\right)$,

$$
\begin{aligned}
\left\langle d^{*} \varphi, d^{*} \varphi\right\rangle & =\sum_{j=1}^{2}\left\|\left(p_{j}-A_{j}\right) \varphi\right\|^{2}+\langle\varphi, B \varphi\rangle, \\
\langle d \varphi, d \varphi\rangle & =\sum_{j=1}^{2}\left\|\left(p_{j}-A_{j}\right) \varphi\right\|^{2}-\langle\varphi, B \varphi\rangle
\end{aligned}
$$

holds. This implies the commutator relation

$$
\left\langle\varphi,\left[d, d^{*}\right] \varphi\right\rangle \stackrel{\text { def }}{=}\left\langle d^{*} \varphi, d^{*} \varphi\right\rangle-\langle d \varphi, d \varphi\rangle=2\langle\varphi, B \varphi\rangle, \quad \varphi \in C_{0}^{\infty}\left(\mathbb{R}^{2} ; \mathbb{C}\right) .
$$

The idea in [14] is to use this commutator to study the essential spectrum of $d d^{*}$ and $d^{*} d$. In order to extend this identity we define these operators as quadratic forms 
and show that $\mathcal{Q}(b) \supset \mathcal{Q}\left(d^{*} d\right)=\mathcal{Q}\left(d d^{*}\right)$ and $|b|^{1 / 2}\left(d^{*} d+1\right)^{-1 / 2}$ is a compact operator. Here $\mathcal{Q}(\cdot)$ is used to denote the form domain.

Let us define

$$
\begin{aligned}
& q_{1}(\varphi, \varphi)=q_{1}[\varphi] \stackrel{\text { def }}{=}\|d \varphi\|^{2}, \\
& q_{2}(\varphi, \varphi)=q_{2}[\varphi] \stackrel{\text { def }}{=}\left\|d^{*} \varphi\right\|^{2},
\end{aligned}
$$

with form domains $\mathcal{Q}\left(q_{1}\right)=\mathscr{D}(d)$ and $\mathcal{Q}\left(q_{2}\right)=\mathscr{D}\left(d^{*}\right)$. Since $d$ and $d^{*}$ are closed (see Subsection 3.3) we have that $q_{1}$ and $q_{2}$ are closed and positive. Thus, associated to $q_{j}, j=1,2$, there is a unique self-adjoint operator $T_{j}$ characterized as follows:

$$
\begin{gathered}
\left\langle\psi, T_{j} \varphi\right\rangle=q_{j}(\psi, \varphi), \quad \psi \in \mathcal{Q}\left(q_{j}\right), \varphi \in \mathcal{D}\left(T_{j}\right), \\
\mathcal{D}\left(T_{j}\right)=\left\{\varphi \in Q\left(q_{j}\right) \mid \exists \eta \in L^{2}\left(\mathbb{R}^{2} ; \mathbb{C}\right), \forall \psi \in \mathcal{C}, q_{j}(\psi, \varphi)=\langle\psi, \eta\rangle\right\},
\end{gathered}
$$

where $\ell$ is any form core of $q_{j}$. It is easy to check using (11) that in fact $T_{1}=d^{*} d$ and $T_{2}=d d^{*}$. Note that since the restrictions of $d$ and $d^{*}$ to $C_{0}^{\infty}\left(\mathbb{R}^{2}, \mathbb{C}\right)$ are closable $C_{0}^{\infty}\left(\mathbb{R}^{2}, \mathbb{C}\right)$ is a form core for $q_{1}$ and $q_{2}$. We define yet another quadratic form. For $\varphi \in C_{0}^{\infty}\left(\mathbb{R}^{2}, \mathbb{C}\right)$ we set

$$
\tilde{q}_{3}[\varphi] \stackrel{\text { def }}{=} \sum_{j=1}^{2}\left\|\left(p_{j}-A_{j}\right) \varphi\right\|^{2} .
$$

It is known (see [27]) that $\tilde{q}_{3}$ is closable and we denote its closure by $q_{3}$. Its associated self-adjoint operator $H_{S} \stackrel{\text { def }}{=}(\mathbf{p}-\mathbf{A})^{2}$ is the usual magnetic Schrödinger operator. Recall that $|b|^{1 / 2}$ is relative $\sqrt{\mathbf{p}^{2}+1}$-compact. Using the diamagnetic inequality for $|\mathbf{p}-\mathbf{A}|$ (see e.g. [11]) and arguing as in [2], Theorem 2.6, we conclude that $\mathcal{Q}\left(q_{3}\right)=\mathscr{D}\left(H_{S}^{1 / 2}\right) \subset \mathscr{D}\left(|b|^{1 / 2}\right)$ and that $|b|^{1 / 2}$ is relative $H_{S}^{1 / 2}$-compact. Thus, the quadratic form

$$
\beta[\varphi] \stackrel{\text { def }}{=} B_{0}\|\varphi\|^{2}+\left\langle\operatorname{sgn}(b)|b|^{1 / 2} \varphi,|b|^{1 / 2} \varphi\right\rangle
$$

is in absolute value bounded with respect to $q_{3}$ with bound 0 . In particular,

$$
q_{3}^{ \pm}[\varphi] \stackrel{\text { def }}{=} q_{3}[\varphi] \pm \beta[\varphi], \quad \varphi \in \mathbb{Q}\left(q_{3}\right),
$$

is closed. Observing that by (9) we have that $q_{2} \uparrow C_{0}^{\infty}=q_{3}^{+} \uparrow C_{0}^{\infty}$ and $q_{1} \uparrow c_{0}^{\infty}=$ $q_{3}^{-} \uparrow C_{0}^{\infty}$ and using that $C_{0}^{\infty}\left(\mathbb{R}^{2} ; \mathbb{C}\right)$ is a form core for $q_{1}, q_{2}, q_{3}$ and $q_{3}^{ \pm}$we conclude that $\mathcal{Q}\left(q_{1}\right)=\mathcal{Q}\left(q_{2}\right)=\mathcal{Q}\left(q_{3}\right) \equiv \mathcal{Q}$ and $q_{1}=q_{3}^{-}$and $q_{2}=q_{3}^{+}$. Moreover,

$$
\begin{aligned}
& d d^{*}=(\mathbf{p}-\mathbf{A})^{2}+B, \\
& d^{*} d=(\mathbf{p}-\mathbf{A})^{2}-B,
\end{aligned}
$$

in the sense of quadratic forms on $\mathcal{Q}$ and hence the commutator formula (10) extends to $\mathcal{Q}$. 
Lemma 1. Let $B=B_{0}+b$ with $B_{0}>0$ and $|b|^{1 / 2} \in L_{\text {loc }}^{2}\left(\mathbb{R}^{2} ; \mathbb{R}\right)$ be relative $\sqrt{\mathbf{p}^{2}+1}$-compact. Let $\mathbf{A} \in L_{\mathrm{loc}}^{p}\left(\mathbb{R}^{2} ; \mathbb{R}^{2}\right), p \in(2, \infty]$, with curl $\mathbf{A}=B$. Then, either one of the following statements holds:

(i) $\sigma_{\text {ess }}\left(d^{*} d\right)=\emptyset$;

(ii) $\sigma_{\text {ess }}\left(d^{*} d\right)=\left\{2 B_{0} n: n \in \mathbb{N}_{0}\right\}$ and $\sigma_{\text {ess }}\left(d d^{*}\right)=\left\{2 B_{0} n: n \in \mathbb{N}\right\}$.

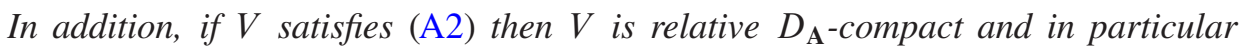
$\sigma_{\text {ess }}\left(D_{\mathbf{A}}\right)=\sigma_{\text {ess }}\left(D_{\mathbf{A}}+V\right)$.

Remark 8. Note that our assumption on $B$ are satisfied if $B$ fulfills (A1). Indeed, in this case $|b|^{1 / 2}\left(\mathbf{p}^{2}+1\right)^{-1 / 2}$ is compact by Lemma 12 in Appendix B.

Moreover, note that if $B \in L_{\mathrm{loc}}^{q}\left(\mathbb{R}^{2} ; \mathbb{R}\right)$ for some $q>1$ we can always find $\mathbf{A} \in L_{\mathrm{loc}}^{p}\left(\mathbb{R}^{2} ; \mathbb{R}^{2}\right)$ for some $p \in(2, \infty]$. In order to see this define $h$ to be a solution of

$$
\Delta h=B .
$$

A local solution to this equation is given by the Newton potential $h_{N}$ of $B$. We know that $h_{N} \in W^{2, q}(\Omega)$ by the Calderón-Zygmund inequality, where $\Omega \subset \mathbb{R}^{2}$ is a bounded domain (see e.g. [12], Section 9.4). This property extends to any solution $h$ of (13) since $h-h_{N}$ is harmonic on $\Omega$. Therefore, $h \in W_{\mathrm{loc}}^{2, q}\left(\mathbb{R}^{2}\right)$. Now one can define $\mathbf{A} \stackrel{\text { def }}{=}\left(-\partial_{2} h, \partial_{1} h\right)$. Clearly, $A_{j} \in W_{\text {loc }}^{1, q}\left(\mathbb{R}^{2}\right)$. By standard Sobolev inequalities one obtains that $A_{j} \in L_{\mathrm{loc}}^{t}\left(\mathbb{R}^{2} ; \mathbb{R}\right)$ for some $2<t<\infty$ if $q \in(1,2]$ and $A_{j} \in L_{\mathrm{loc}}^{\infty}\left(\mathbb{R}^{2} ; \mathbb{R}\right)$ if $q>2$.

Proof. First note that for any $\lambda \geq 0$ the operator $\left(d^{*} d+2 B_{0}+\lambda\right)^{-1 / 2}$ maps $L^{2}\left(\mathbb{R}^{2} ; \mathbb{C}\right)$ onto $\mathscr{D}\left(\sqrt{d^{*} d}\right)$ which equals $\mathcal{Q}$ and $\mathscr{D}\left(H_{S}^{1 / 2}\right)$. Thus, by the closed graph theorem, the operator $\left(H_{S}+1\right)^{1 / 2}\left(d^{*} d+2 B_{0}+\lambda\right)^{-1 / 2}$ is bounded. In particular,

$$
\begin{aligned}
& |b|^{1 / 2}\left(d^{*} d+2 B_{0}+\lambda\right)^{-1 / 2} \\
& \quad=|b|^{1 / 2}\left(H_{S}+1\right)^{-1 / 2}\left(H_{S}+1\right)^{1 / 2}\left(d^{*} d+2 B_{0}+\lambda\right)^{-1 / 2}
\end{aligned}
$$

is compact. Hence, the operator

$$
T(\lambda) \stackrel{\text { def }}{=} \overline{\left(d^{*} d+2 B_{0}+\lambda\right)^{-1 / 2} \operatorname{sgn}(b)|b|^{1 / 2}}|b|^{1 / 2}\left(d^{*} d+2 B_{0}+\lambda\right)^{-1 / 2}
$$

is also compact. It is easy to see that $\lambda>0$ can be chosen so large that $\|T(\lambda)\|<1$. For such $\lambda$ 's we have, according to the resolvent formula for operators defined as quadratic forms (see [26]), that

$$
\begin{aligned}
\left(d^{*} d\right. & \left.+2 B_{0}+2 b+\lambda\right)^{-1} \\
& =\left(d^{*} d+2 B_{0}+\lambda\right)^{-1 / 2}(1+T(\lambda))^{-1}\left(d^{*} d+2 B_{0}+\lambda\right)^{-1 / 2} .
\end{aligned}
$$

Note that the inverse of $1+T(\lambda)$ is well defined as a geometric expansion. Since $(1+T(\lambda))^{-1}-1$ is compact, we conclude that the resolvent difference between 
$d^{*} d+2 B_{0}+2 b+\lambda$ and $d^{*} d+2 B_{0}+\lambda$ is also compact. Therefore, by Weyl's theorem, the two operators have the same essential spectrum. Using this and (12) we deduce that

$$
\sigma_{\mathrm{ess}}\left(d d^{*}\right)=\sigma_{\mathrm{ess}}\left(d^{*} d+2 B_{0}+2 b\right)=\sigma_{\mathrm{ess}}\left(d^{*} d+2 B_{0}\right) .
$$

The latter equality and Equation (7) imply (here we follow [14])

$$
\begin{aligned}
S & \stackrel{\text { def }}{=} \sigma_{\text {ess }}\left(d^{*} d\right), \quad S \subset[0, \infty), \\
S \backslash\{0\} & =S+2 B_{0} .
\end{aligned}
$$

Assume now that $S \neq \emptyset$, then it is easy to see from (15) that $0 \in S$ and hence $2 B_{0} n \in S, n \in \mathbb{N}_{0}$. Note also that no other points can belong to $S$. Hence, using (14) we get that $\sigma_{\text {ess }}\left(d d^{*}\right)=2 B_{0} n, n \in \mathbb{N}$.

Now, assume that $V$ fulfills (A2). Then, $V$ is relative $\sqrt{\mathbf{p}^{2}+1}$-compact (see Lemma 12 in Appendix B). It follows by the diamagnetic inequality that $V$ is relative $H_{S}^{1 / 2}$-compact and consequently (arguing as before for $\left.b\right) \mathscr{D}(V) \supset \mathcal{Q}$ and the operators $V\left(d d^{*}+\lambda^{2}\right)^{-1 / 2}$ and $V\left(d^{*} d+\lambda^{2}\right)^{-1 / 2}$ are compact for any $\lambda \neq 0$. From these considerations follow that $V\left(D_{\mathbf{A}}-\mathrm{i} \lambda\right)^{-1}$ is compact, since the identity

$$
\begin{aligned}
& \left(D_{\mathbf{A}}-\mathrm{i} \lambda\right)^{-1} \\
& \quad=\left(D_{\mathbf{A}}^{2}+\lambda^{2}\right)^{-1 / 2}\left[\left(D_{\mathbf{A}}^{2}+\lambda^{2}\right)^{-1 / 2}\left(D_{\mathbf{A}}+\mathrm{i} \lambda\right)\right] \\
& \quad=\left(\begin{array}{cc}
\left(d^{*} d+\lambda^{2}\right)^{-1 / 2} & 0 \\
0 & \left(d d^{*}+\lambda^{2}\right)^{-1 / 2}
\end{array}\right) \cdot\left[\left(D_{\mathbf{A}}^{2}+\lambda^{2}\right)^{-1 / 2}\left(D_{\mathbf{A}}+\mathrm{i} \lambda\right)\right]
\end{aligned}
$$

holds and the operator in $[\ldots]$ is bounded. Therefore,

$$
\sigma_{\mathrm{ess}}\left(D_{\mathbf{A}}+V\right)=\sigma_{\mathrm{ess}}\left(D_{\mathbf{A}}\right) .
$$

We note that if $b$ satisfies (A1) then $\operatorname{Ker}\left(d^{*} d\right)$ is infinitely degenerated. Indeed, this follows from the fact that

$$
\begin{aligned}
& \int_{\mathbb{R}^{2}}[B]_{+} d^{2} x=\infty, \\
& \int_{\mathbb{R}^{2}}[B]_{-} d^{2} x<\infty,
\end{aligned}
$$

(where $[f]_{+}$and $[f]_{-}$are the positive and negative parts of $f$ ) which shows that $B=B_{0}+b$ fulfills the conditions of [24], Corollary 3.4. In particular, we know that

$$
\operatorname{Ker}\left(d^{*} d\right)=\left\{\omega e^{-h} \mid \omega e^{-h} \in L^{2}\left(\mathbb{R}^{2} ; \mathbb{C}\right), \omega \text { is analytic in } x_{1}+\mathrm{i} x_{2}\right\},
$$

where $h$ is a solution of the equation $\Delta h=B$; see [24]. Therefore, we get the following result. 
Lemma 2. Assume that $B$ satisfies (A1) and let $\mathbf{A} \in L_{\mathrm{loc}}^{p}\left(\mathbb{R}^{2} ; \mathbb{R}^{2}\right), p \in(2, \infty]$, with $\operatorname{curl} \mathbf{A}=B$. Then,

$$
\begin{aligned}
& \sigma_{\text {ess }}\left(d^{*} d\right)=\left\{2 B_{0} n \mid n \in \mathbb{N}_{0}\right\}, \\
& \sigma_{\text {ess }}\left(d d^{*}\right)=\left\{2 B_{0} n \mid n \in \mathbb{N}\right\} .
\end{aligned}
$$

In particular,

$$
\sigma_{\text {ess }}\left(D_{\mathbf{A}}\right)=\sigma_{\text {ess }}\left(D_{\mathbf{A}_{0}}\right)=\left\{l_{n} \mid n \in \mathbb{Z}\right\} .
$$

Moreover, 0 is an isolated point of $\sigma\left(D_{\mathbf{A}}\right)$ and $\sigma\left(d^{*} d\right)$.

Proof. Due to our previous discussion we see that $0 \in \sigma_{\text {ess }}\left(d^{*} d\right)$. This combined with Lemma 1 imply (16). That 0 is an isolated point of $\sigma\left(d^{*} d\right)$ follows by noting that, since $0 \notin \sigma_{\text {ess }}\left(d d^{*}\right), 0$ is neither an accumulation point of $\sigma\left(d d^{*}\right)$ nor of $\sigma\left(d^{*} d\right)$. The statements on $\sigma\left(D_{\mathbf{A}}\right)$ are now a consequence of Proposition 1.

Lemma 3. Assume that $B$ satisfies (A1) and let $\mathbf{A} \in L_{\mathrm{loc}}^{p}\left(\mathbb{R}^{2} ; \mathbb{R}^{2}\right), p \in(2, \infty]$, with $\operatorname{curl} \mathbf{A}=B$. Then, we have

(a) If $b \leq 0$ and strictly negative on some open set, then

$$
\operatorname{dim}\left(\operatorname{Ran}\left(\mathbb{1}_{\left(0, \sqrt{2 B_{0}}\right)}\left(D_{\mathbf{A}}\right)\right)\right)=\operatorname{dim}\left(\operatorname{Ran}\left(\mathbb{1}_{\left(-\sqrt{2 B_{0}}, 0\right)}\left(D_{\mathbf{A}}\right)\right)\right)=\infty .
$$

(b) If $b \geq 0$ then

$$
\operatorname{dim}\left(\operatorname{Ran}\left(\mathbb{1}_{\left(0, \sqrt{2 B_{0}}\right)}\left(D_{\mathbf{A}}\right)\right)\right)=\operatorname{dim}\left(\operatorname{Ran}\left(\mathbb{1}_{\left(-\sqrt{2 B_{0}}, 0\right)}\left(D_{\mathbf{A}}\right)\right)\right)=0 .
$$

Proof. We may choose $\mathbf{A} \stackrel{\text { def }}{=}\left(-\partial_{2} h, \partial_{1} h\right)$ where $h$ is a solution of $\Delta h=B$. Due to Remark 8 we know that $\mathbf{A} \in L_{\text {loc }}^{p}\left(\mathbb{R}^{2}, \mathbb{R}^{2}\right)$ for some $p>2$.

Part (a). Let $\Omega$ be an open set with $b \nmid \Omega<0$. Recall that there are infinitely many functions $\omega$, analytic in $x_{1}+\mathrm{i} x_{2}$, with $\psi \stackrel{\text { def }}{=} \omega e^{-h} \in \operatorname{Ker}\left(d^{*} d\right)$. For such $\psi$ we have, using (12),

$$
\left\langle\psi, d d^{*} \psi\right\rangle=2\langle\psi, B \psi\rangle \leq 2 B_{0}\|\psi\|^{2}+2 \int_{\Omega} b(\mathbf{x})|\psi(\mathbf{x})|^{2} d \mathbf{x}<2 B_{0}\|\psi\|^{2},
$$

where in the last inequality we use the fact that $\psi$ can not vanish on $\Omega$. Let $\left(\psi_{n}\right)_{n \in \mathbb{N}}$ be an orthonormal system such that $\psi_{n} \stackrel{\text { def }}{=} e^{-h} \omega_{n} \in \operatorname{Ker} d^{*} d$ with $\omega_{n}$ analytic in $x_{1}+\mathrm{i} x_{2}$. For $N \in \mathbb{N}$ define the self-adjoint matrix $M_{N} \stackrel{\text { def }}{=}\left(\left\langle\psi_{n}, d d^{*} \psi_{m}\right\rangle\right)_{1 \leq n, m \leq N}$. It follows from (17) that $M_{N}<2 B_{0}$. The Rayleigh-Ritz principle implies

$$
0 \leq \mu_{n}\left(d d^{*}\right) \leq \mu_{n}\left(M_{N}\right)<2 B_{0}, \quad n=1, \ldots, N,
$$


where we write

$$
\mu_{n}(T) \stackrel{\text { def }}{=} \sup _{\varphi_{1}, \ldots, \varphi_{n-1}} \inf _{\substack{\psi \operatorname{span}\left\{\varphi_{1}, \ldots, \varphi_{n-1}\right\}^{\perp} \\\|\psi\|=1, \psi \in Q(T)}}\langle\psi, T \psi\rangle
$$

for some self-adjoint operator T. Since $N$ is arbitrary the mini-max principle implies that $\operatorname{dim}\left(\operatorname{Ran}\left(\mathbb{1}_{\left[0, \sqrt{2 B_{0}}\right)}\left(d d^{*}\right)\right)\right)=\infty$. It follows that $\operatorname{dim}\left(\operatorname{Ran}\left(\mathbb{1}_{\left(0, \sqrt{2 B_{0}}\right)}\left(d d^{*}\right)\right)\right)=$ $\infty$, for $0 \notin \sigma_{\text {ess }}\left(d d^{*}\right)$ by Lemma 2 . The claim is now a consequence of Proposition 1 and (7).

Part (b). In this case we have that $d d^{*} \geq 2 B_{0}$, since $d d^{*}-d^{*} d=2 B \geq 2 B_{0}$. Thus, the claim follows now from Proposition 1 and (7).

\section{Super-exponential localization}

The proof of Theorem 2 follows the ideas developed in [6]. An essential ingredient is that, by means of suitable local gauge transformations on certain regions outside a big ball of radius $n$ centered at the origin, one can replace the operator $D_{\mathbf{A}}$ by a Dirac operator $D_{\mathbf{A}_{n}}$ with $\mathbf{A}_{n}=\mathbf{A}_{0}+\mathbf{a}^{n}$, where $\mathbf{a}^{n}$ is a magnetic vector potential of a magnetic field $b_{n}$ satisfying $\lim _{n \rightarrow \infty}\left\|b_{n}\right\|_{\infty}=0$. The advantage of this is that we can obtain explicit $L^{p}$ estimates (see Lemma 4 below) for the resolvents of $D_{\mathbf{A}_{n}}$, conjugated with exponential weights. These estimates can be derived using a certain resolvent expansion, see (35), in combination with an explicit expression for the Green kernel of $D_{\mathbf{A}_{0}}$ that can be found in Appendix A below.

Before stating these $L^{p}$ estimates let us fix some notation. For $p, q \in[1, \infty]$ we denote by $\mathcal{B}(p, q)$ the space of bounded operators from $L^{p}\left(\mathbb{R}^{2} ; \mathbb{C}^{2}\right)$ to $L^{q}\left(\mathbb{R}^{2} ; \mathbb{C}^{2}\right)$ and write, for $T \in \mathscr{B}(p, q)$,

$$
\|T\|_{p, q} \stackrel{\text { def }}{=}\|T\|_{\mathcal{B}(p, q)} .
$$

Let $\gamma \geq 0$ and $\mathbf{u} \in \mathbb{R}^{2}$ with $|\mathbf{u}|=1$. We define the exponential weight function as

$$
F(\mathbf{x}) \stackrel{\text { def }}{=} \gamma \mathbf{u} \cdot \mathbf{x}, \quad \mathbf{x} \in \mathbb{R}^{2} .
$$

Let $b_{n}$ be a magnetic field with $\lim _{n \rightarrow \infty}\left\|b_{n}\right\|_{\infty}=0$ and $\mathbf{a}^{n}$ be the associated vector potential in the transversal gauge, i.e.,

$$
\mathbf{a}^{n}(\mathbf{x}) \stackrel{\text { def }}{=} \int_{0}^{1} b_{n}(s \mathbf{x}) \wedge \mathbf{x} s d s,
$$

where we write $a \wedge \mathbf{v} \stackrel{\text { def }}{=} a\left(-v_{2}, v_{1}\right)$ for $a \in \mathbb{R}$ and $\mathbf{v} \in \mathbb{R}^{2}$. The proof of the lemma below can be found at the end of this section. 
Lemma 4. Let $V_{n} \in L^{\infty}\left(\mathbb{R}^{2} ; \mathbb{R}\right), n \in \mathbb{N}$, be a family of electric potentials satisfying

$$
\lim _{n \rightarrow \infty}\left\|V_{n}\right\|_{\infty}=0 \text {. }
$$

For any $n \in \mathbb{N}$ define the family of self-adjoint operators $D_{\mathbf{A}_{n}}+V_{n}$, where $\mathbf{A}_{n} \stackrel{\text { def }}{=}$ $\mathbf{A}_{0}+\mathbf{a}^{n}$ and $\mathbf{a}^{n}$ is given in (19). Let $z \in \mathbb{R} \backslash \sigma\left(D_{\mathbf{A}_{0}}\right)$ and $q, r \in[1, \infty]$ be such that $1+\frac{1}{r}-\frac{1}{q}=\frac{1}{p}$ for some $p \in[1,2)$. Then, there exists $N>0$ such that, for all $n>N, z \notin \sigma\left(D_{\mathbf{A}_{n}}+V_{n}\right)$ and

$$
e^{F}\left(D_{\mathbf{A}_{n}}+V_{n}-z\right)^{-1} e^{-F} \in \mathscr{B}(q, r) .
$$

In what follows we apply the above result to show Theorem 2.

Proof of Theorem 2. For $n \in \mathbb{N}$ and $\mathbf{u} \in \mathbb{R}^{2}$ with $|\mathbf{u}|=1$ set

$$
\Omega_{n}=\left\{\mathbf{x} \in \mathbb{R}^{2}: \mathbf{u} \cdot \mathbf{x}>n\right\} .
$$

For $j \in\{1,2,3\}$ define $\chi_{j} \in C^{\infty}\left(\mathbb{R}^{2} ;[0,1]\right)$ with $\chi_{j}=0$ on $\mathbb{R}^{2} \backslash \Omega_{j n}$ and $\chi_{j}=1$ on $\Omega_{(j+1) n}$. We choose $n$ so large that

$$
\|b\|_{L^{\infty}\left(\Omega_{n}\right)}<\infty
$$

Since $b \in L_{\mathrm{loc}}^{q}\left(\mathbb{R}^{2}\right), q>1$, we find a vector potential $\mathbf{a} \in L_{\mathrm{loc}}^{p}\left(\mathbb{R}^{2} ; \mathbb{R}^{2}\right), p>2$, with curl $\mathbf{a}=b$ (see Remark 8). Define, for $\mathbf{x} \in \mathbb{R}^{2}$,

$$
\mathbf{a}^{n}(\mathbf{x})=\int_{0}^{1} b_{n}(s \mathbf{x}) \wedge \mathbf{x} s d s
$$

where $b_{n} \stackrel{\text { def }}{=} \mathbb{1}_{\Omega_{n}} b \in L^{\infty}\left(\mathbb{R}^{2}\right)$. Observe that

$$
\operatorname{curl} \mathbf{a}=\operatorname{curl} \mathbf{a}^{n} \quad \text { on } \Omega_{n},
$$

that $\Omega_{n}$ is simply connected, and that $\mathbf{a}^{n}, \mathbf{a} \in L_{\text {loc }}^{p}\left(\mathbb{R}^{2} ; \mathbb{R}^{2}\right)$ for some $p>2$. Therefore, there exists a gauge function $\tilde{\Phi}_{n} \in W_{\text {loc }}^{1, p}\left(\Omega_{n}\right)$ such that (see [17], Lemma 1.1)

$$
\nabla \tilde{\Phi}_{n}=\mathbf{a}-\mathbf{a}^{n} \quad \text { on } \Omega_{n} .
$$

By multiplying $\tilde{\Phi}_{n}$ with a $C^{\infty}$ - cutoff function we may define a $\Phi_{n} \in W_{\text {loc }}^{1, p}\left(\mathbb{R}^{2}\right)$ that coincides with $\tilde{\Phi}_{n}$ on $\Omega_{2 n}$. In particular, we find that

$$
\nabla \Phi_{n}=\mathbf{a}-\mathbf{a}^{n} \quad \text { on } \Omega_{2 n} .
$$

Define now $V_{n} \stackrel{\text { def }}{=} \chi_{1} V$ and observe that $\left\|V_{n}\right\|_{\infty} \rightarrow 0$ as $n \rightarrow \infty$. Then we get, for any $\eta \in \mathcal{C}_{0}^{\infty}\left(\mathbb{R}^{2} ; \mathbb{C}^{2}\right)$ and $j \in\{2,3\}$, using (22) and the identity $\chi_{j}=\chi_{1} \chi_{j}$,

$$
\begin{aligned}
\chi_{j}\left(D_{\mathbf{A}}+V\right) \eta & =\chi_{j}\left(D_{\mathbf{A}_{0}}-\sigma \cdot \mathbf{a}+V_{n}\right) \eta \\
& =\chi_{j}\left(D_{\mathbf{A}_{0}}-\sigma \cdot \nabla \Phi_{n}-\sigma \cdot \mathbf{a}^{n}+V_{n}\right) \eta \\
& =\chi_{j} e^{\mathrm{i} \Phi_{n}}\left(D_{\mathbf{A}_{0}}-\sigma \cdot \mathbf{a}^{n}+V_{n}\right) e^{-\mathrm{i} \Phi_{n}} \eta .
\end{aligned}
$$


Set $\mathbf{A}_{n} \stackrel{\text { def }}{=} \mathbf{A}_{0}+\mathbf{a}^{n}$ and let $\Psi$ be an eigenfunction of $D_{\mathbf{A}}$ with eigenvalue $E \notin$ $\sigma\left(D_{\mathbf{A}_{0}}\right)$. By the previous computation we obtain, for any $\eta \in \mathcal{C}_{0}^{\infty}\left(\mathbb{R}^{2} ; \mathbb{C}^{2}\right)$ and $j \in\{2,3\}$,

$$
\begin{aligned}
\left\langle e^{\mathrm{i} \Phi_{n}}\left(D_{\mathbf{A}_{n}}+V_{n}-E\right) e^{-\mathrm{i} \Phi_{n}} \eta \mid \chi_{j} \Psi\right\rangle & =\left\langle\left(D_{\mathbf{A}}+V-E\right) \eta \mid \chi_{j} \Psi\right\rangle \\
& =\left\langle\mathrm{i} \boldsymbol{\sigma} \cdot \nabla \chi_{j} \eta \mid \Psi\right\rangle .
\end{aligned}
$$

This equality extends to any $\eta$ in the domain of $e^{\mathrm{i} \Phi_{n}}\left(D_{\mathbf{A}_{n}}+V_{n}-E\right) e^{-\mathrm{i} \Phi_{n}}$, since $\mathcal{C}_{0}^{\infty}\left(\mathbb{R}^{2} ; \mathbb{C}^{2}\right)$ is a core for $e^{\mathrm{i} \Phi_{n}}\left(D_{\mathbf{A}_{n}}+V_{n}-E\right) e^{-\mathrm{i} \Phi_{n}} ;$ Subsection 3.2. Clearly, $\mathbf{A}_{n}$ and $V_{n}$ satisfy the assumptions of Lemma 4 . Thus, for $n$ sufficiently large, $E \notin \sigma\left(D_{\mathbf{A}_{n}}\right)$ and we may replace $\eta$ by $e^{\mathrm{i} \Phi_{n}}\left(D_{\mathbf{A}_{n}}+V_{n}-E\right)^{-1} e^{-\mathrm{i} \Phi_{n}} \eta$ obtaining that

$$
\chi_{j} \Psi=-\mathrm{i} e^{\mathrm{i} \Phi_{n}}\left(D_{\mathbf{A}_{n}}+V_{n}-E\right)^{-1} e^{-\mathrm{i} \Phi_{n}}\left(\sigma \cdot \nabla \chi_{j}\right) \Psi, \quad j \in\{2,3\} .
$$

Observe that using (23) for $j=2$ in combination with Lemma 4, with $q=2, r=3$, and $F=0$, we obtain that

$$
\chi_{2} \Psi \in L^{3}\left(\mathbb{R}^{2} ; \mathbb{C}^{2}\right) .
$$

We use again (23), for large $n$, to get in addition that

$$
e^{F} \chi_{3} \Psi=-\mathrm{i} e^{\mathrm{i} \Phi_{n}}\left(e^{F}\left(D_{\mathbf{A}_{n}}+V_{n}-E\right)^{-1} e^{-F}\right)\left(e^{-\mathrm{i} \Phi_{n}} e^{F}\left(\sigma \nabla \chi_{3}\right) \chi_{2} \Psi\right) .
$$

Since $\operatorname{supp}\left(\nabla \chi_{3}\right) \subset \Omega_{3 n} \backslash \Omega_{4 n}$ we find thanks to (24) that $e^{-\mathrm{i} \Phi_{n}} e^{F}\left(\sigma \nabla \chi_{3}\right) \chi_{2} \Psi \in$ $L^{2}\left(\mathbb{R}^{2} ; \mathbb{C}^{2}\right) \cap L^{3}\left(\mathbb{R}^{2} ; \mathbb{C}^{2}\right)$. Thus, we may apply Lemma 4 with $q=3, r=\infty$ and $q=2, r=2$ to obtain the decay in the $L^{\infty}$ and $L^{2}$ norms respectively for $n \geq n_{0}$ sufficiently large. We obtain the desired bound (5) from (25) by varying $F$ over finitely many vectors $\mathbf{u}$.

Proof of Lemma 4. Recall that the magnetic vector potential is given by $\mathbf{A}_{n}=\mathbf{A}_{0}+$ $\mathbf{a}^{n}$ where $\mathbf{a}^{n}$ is defined in (19).

A simple calculation shows that the vector potential

$$
\mathbf{a}_{\mathbf{x}^{\prime}}^{n}(\mathbf{x})=\int_{0}^{1} b_{n}\left(\mathbf{x}^{\prime}+s\left(\mathbf{x}-\mathbf{x}^{\prime}\right)\right) \wedge\left(\mathbf{x}-\mathbf{x}^{\prime}\right) s d s, \quad \mathbf{x}^{\prime} \in \mathbb{R}^{2},
$$

is also a vector potential of the magnetic field $b_{n}$. A crucial property of $\mathbf{a}_{\mathbf{x}^{\prime}}^{n}$ is that

$$
\left|\mathbf{a}_{\mathbf{x}^{\prime}}^{n}(\mathbf{x})\right| \leq\left\|b_{n}\right\|_{\infty} \cdot\left|\mathbf{x}-\mathbf{x}^{\prime}\right|, \quad \mathbf{x}, \mathbf{x}^{\prime} \in \mathbb{R}^{2} .
$$

Since curl $\mathbf{a}_{\mathbf{x}^{\prime}}^{\mathrm{n}}=\operatorname{curl} \mathbf{a}^{\mathrm{n}}$ there exists a function $\varphi_{n}: \mathbb{R}^{2} \rightarrow \mathbb{R}$ with

$$
\nabla_{\mathbf{x}} \varphi_{n}\left(\mathbf{x}, \mathbf{x}^{\prime}\right)=\mathbf{a}^{n}(\mathbf{x})-\mathbf{a}_{\mathbf{x}^{\prime}}^{n}(\mathbf{x}) .
$$

We may further require that

$$
\varphi_{n}(\mathbf{x}, \mathbf{x})=0 .
$$


The proof of Lemma 4 is based upon $L^{p}$ estimates for the resolvent expansion (35) below. We start by defining the relevant objects and list their $L^{p}$ properties. For $z \in \mathbb{R} \backslash \sigma\left(D_{\mathbf{A}_{0}}\right)$ let $G_{0}\left(\mathbf{x}, \mathbf{x}^{\prime}, z\right)$ be a representation of the Green kernel of $\left(D_{\mathbf{A}_{0}}-z\right)^{-1}$ as $2 \times 2$-matrix. Due to (60) from Appendix A and the triangular inequality we obtain that

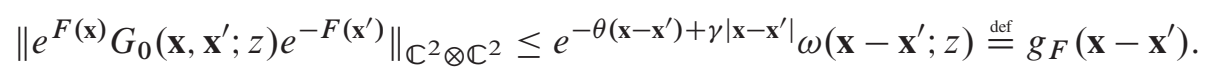

We observe that thanks to (61) we have that

$$
\begin{cases}g_{F} \in L^{t}\left(\mathbb{R}^{2}\right) & t \in[1,2), \\ |\mathbf{x}| g_{F} \in L^{t}\left(\mathbb{R}^{2}\right) & t \in[1, \infty] .\end{cases}
$$

We introduce, for $n \in \mathbb{N}$, the integral operators

$$
S_{n}(z), T_{n}(z): L^{2}\left(\mathbb{R}^{2} ; \mathbb{C}^{2}\right) \longrightarrow L^{2}\left(\mathbb{R}^{2} ; \mathbb{C}^{2}\right)
$$

with

$$
\begin{aligned}
& \left(S_{n}(z) f\right)(\mathbf{x}) \stackrel{\text { def }}{=} \int_{\mathbb{R}^{2}} e^{\mathrm{i} \varphi_{n}\left(\mathbf{x}, \mathbf{x}^{\prime}\right)} G_{0}\left(\mathbf{x}, \mathbf{x}^{\prime} ; z\right) f\left(\mathbf{x}^{\prime}\right) d \mathbf{x}^{\prime}, \\
& \left(T_{n}(z) f\right)(\mathbf{x}) \stackrel{\text { def }}{=} \int_{\mathbb{R}^{2}} \sigma \cdot a_{\mathbf{x}^{\prime}}^{n}(\mathbf{x}) e^{\mathrm{i} \varphi_{n}\left(\mathbf{x}, \mathbf{x}^{\prime}\right)} G_{0}\left(\mathbf{x}, \mathbf{x}^{\prime} ; z\right) f\left(\mathbf{x}^{\prime}\right) d \mathbf{x}^{\prime},
\end{aligned}
$$

where $\varphi_{n}$ is determined by (28) and (29). Notice that in view of (30), (27), and Young's inequality (see [18], Section 4.2) both operators are well defined and bounded. In fact, since

$$
\left\|\left(e^{F} T_{n}(z) e^{-F}\right)\left(\mathbf{x}, \mathbf{x}^{\prime}\right)\right\|_{\mathbb{C}^{2} \otimes \mathbb{C}^{2}} \leq\left\|b_{n}\right\|_{\infty}\left|\mathbf{x}-\mathbf{x}^{\prime}\right| g_{F}\left(\mathbf{x}-\mathbf{x}^{\prime}\right),
$$

we find by (30) and Young's inequality that, for $q \in[1, \infty]$,

$$
\lim _{n \rightarrow \infty}\left\|e^{F} T_{n}(z) e^{-F}\right\|_{q, q}=0 .
$$

Furthermore, a similar argument implies that, for $q, r \in[1, \infty]$ and $t \in[1,2)$ with $\frac{1}{t}=1+\frac{1}{r}-\frac{1}{q}$,

$$
\sup _{n \in \mathbb{N}}\left\|e^{F} S_{n}(z) e^{-F}\right\|_{q, r}<\infty .
$$

Our next task is to show the following resolvent formula in $L^{2}\left(\mathbb{R}^{2} ; \mathbb{C}^{2}\right)$, for $n \in \mathbb{N}$ so large that $\left\|T_{n}(z)\right\|_{2,2}<1$, see (33),

$$
\left(D_{\mathbf{A}_{n}}-z\right)^{-1}=S_{n}(z) \sum_{k=0}^{\infty} T_{n}(z)^{k} .
$$


Pick functions $f \in L^{2}\left(\mathbb{R}^{2} ; \mathbb{C}^{2}\right)$ and $g \in C_{0}^{\infty}\left(\mathbb{R}^{2} ; \mathbb{C}^{2}\right)$ and, we find

$$
\begin{aligned}
& \left\langle\left(D_{\mathbf{A}_{n}}-z\right) g, S_{n}(z) f\right\rangle \\
& \quad=\int_{\mathbb{R}^{2}}\left\langle\left[\left(D_{\mathbf{A}_{n}}-z\right) g\right](\mathbf{x}), \int_{\mathbb{R}^{2}} e^{\mathrm{i} \varphi_{n}\left(\mathbf{x}, \mathbf{x}^{\prime}\right)} G_{0}\left(\mathbf{x}, \mathbf{x}^{\prime} ; z\right) f\left(\mathbf{x}^{\prime}\right) d \mathbf{x}^{\prime}\right\rangle_{\mathbb{C}^{2}} d \mathbf{x} \\
& \quad=\int_{\mathbb{R}^{2}} \int_{\mathbb{R}^{2}}\left\langle e^{-\mathrm{i} \varphi_{n}\left(\mathbf{x}, \mathbf{x}^{\prime}\right)}\left[\left(D_{\mathbf{A}_{n}}-z\right) g\right](\mathbf{x}), G_{0}\left(\mathbf{x}, \mathbf{x}^{\prime} ; z\right) f\left(\mathbf{x}^{\prime}\right)\right\rangle_{\mathbb{C}^{2}} d \mathbf{x} d \mathbf{x}^{\prime},
\end{aligned}
$$

where Young's inequality together with Lemma 11 from Appendix A enabled us to use Fubini's theorem in the last equality. Observe that due to (28)

$$
e^{-\mathrm{i} \varphi_{n}\left(\mathbf{x}, \mathbf{x}^{\prime}\right)}\left[\left(D_{\mathbf{A}_{n}}-z\right) g\right](\mathbf{x})=\left[\left(D_{\mathbf{A}_{0}}-\sigma \cdot \mathbf{a}_{\mathbf{x}^{\prime}}^{n}-z\right) e^{-\mathrm{i} \varphi_{n}\left(\cdot, \mathbf{x}^{\prime}\right)} g\right](\mathbf{x}) .
$$

Hence, using again Fubini's theorem,

$$
\begin{aligned}
& \left\langle\left(D_{\mathbf{A}_{n}}-z\right) g, S_{n}(z) f\right\rangle= \\
& \quad \int_{\mathbb{R}^{2}}\left\langle\int_{\mathbb{R}^{2}} G_{0}\left(\mathbf{x}^{\prime}, \mathbf{x} ; z\right)\left[\left(D_{\mathbf{A}_{0}}-z\right) e^{-\mathrm{i} \varphi_{n}\left(\cdot, \mathbf{x}^{\prime}\right)} g\right](\mathbf{x}) d \mathbf{x},\left.f\left(\mathbf{x}^{\prime}\right)\right|_{\mathbb{C}^{2}} d \mathbf{x}^{\prime}\right. \\
& \quad-\int_{\mathbb{R}^{2}} \int_{\mathbb{R}^{2}}\left\langle\boldsymbol{\sigma} \cdot \mathbf{a}_{\mathbf{x}^{\prime}}^{n}(\mathbf{x}) e^{-\mathrm{i} \varphi_{n}\left(\mathbf{x}, \mathbf{x}^{\prime}\right)} g(\mathbf{x}), G_{0}\left(\mathbf{x}, \mathbf{x}^{\prime} ; z\right) f\left(\mathbf{x}^{\prime}\right)\right\rangle_{\mathbb{C}^{2}} d \mathbf{x}^{\prime} d \mathbf{x} \\
& =\langle g, f\rangle-\left\langle g, T_{n}(z) f\right\rangle .
\end{aligned}
$$

For the first integral after the first equality above we used (29) together with the fact that $G_{0}$ is the Green kernel of $D_{\mathbf{A}_{0}}$ and thus, for any $\tilde{g} \in C_{0}^{\infty}\left(\mathbb{R}^{2} ; \mathbb{C}^{2}\right)$,

$$
\int_{\mathbb{R}^{2}} G_{0}\left(\mathbf{x}^{\prime}, \mathbf{x} ; z\right)\left[\left(D_{\mathbf{A}_{0}}-z\right) \tilde{g}\right](\mathbf{x}) d \mathbf{x}=\tilde{g}\left(\mathbf{x}^{\prime}\right) \quad \text { a.e. }
$$

Now, since $D_{\mathbf{A}_{n}}$ is essentially self-adjoint on $C_{0}^{\infty}\left(\mathbb{R}^{2} ; \mathbb{C}^{2}\right)$ we can extend the identity (36) for all $g \in \mathscr{D}\left(D_{\mathbf{A}_{n}}\right)$. From this extension follows that $S_{n}(z)$ maps $L^{2}\left(\mathbb{R}^{2} ; \mathbb{C}^{2}\right)$ in $\mathscr{D}\left(D_{\mathbf{A}_{n}}\right)$ and

$$
\left(D_{\mathbf{A}_{n}}-z\right) S_{n}(z) f=f-T_{n}(z) f, \quad f \in L^{2}\left(\mathbb{R}^{2} ; \mathbb{C}^{2}\right) .
$$

This yields, for $n$ large enough, the operator identity

$$
S_{n}(z)=\left(D_{\mathbf{A}_{n}}-z\right)^{-1}\left(1-T_{n}(z)\right),
$$

from which follows (35).

We observe that (20), for $V_{n}=0$, is a consequence of (33), (34), and

$$
\left\|e^{F}\left(D_{\mathbf{A}_{n}}-z\right)^{-1} e^{-F}\right\|_{q, r} \leq\left\|e^{F} S_{n}(z) e^{-F}\right\|_{q, r} \cdot \sum_{k=0}^{\infty}\left(\left\|e^{F} T_{n}(z) e^{-F}\right\|_{q, q}\right)^{k} .
$$


Note that the last sum above converges for $n$ large enough due to (33).

In order to show (20) for $V_{n} \neq 0$ we note that by Hölder's inequality

$$
\left\|V_{n} e^{F}\left(D_{\mathbf{A}_{n}}-z\right)^{-1} e^{-F}\right\|_{q, q} \leq\left\|V_{n}\right\|_{\infty}\left\|e^{F}\left(D_{\mathbf{A}_{n}}-z\right)^{-1} e^{-F}\right\|_{q, q} \longrightarrow 0,
$$

as $n \rightarrow \infty$. Therefore, the following computation is meaningful for $n$ large enough

$$
\begin{aligned}
& \left\|e^{F}\left(D_{\mathbf{A}_{n}}+V_{n}-z\right)^{-1} e^{-F}\right\|_{q, r} \\
& \quad=\left\|e^{F}\left(D_{\mathbf{A}_{n}}-z\right)^{-1}\left(1+V_{n}\left(D_{\mathbf{A}_{n}}-z\right)^{-1}\right)^{-1} e^{-F}\right\|_{q, r} \\
& \quad \leq\left\|e^{F}\left(D_{\mathbf{A}_{n}}-z\right)^{-1} e^{-F}\right\|_{q, r} \sum_{m=0}^{\infty}\left\|\left\{V_{n} e^{F}\left(D_{\mathbf{A}_{n}}-z\right)^{-1} e^{-F}\right\}^{m}\right\|_{q, q} .
\end{aligned}
$$

This finishes the proof.

\section{Gaussian-localization}

In this section we show Theorem 3 on Gaussian localization of eigenfunctions with energies in the discrete spectrum of

$$
H=D_{\mathbf{A}}+V,
$$

under the assumptions (A1)-(A5) stated in the introduction. We choose the magnetic vector potential to be given by

$$
\mathbf{A}(\mathbf{x}) \stackrel{\text { def }}{=} r^{-1} A(r)\left(\begin{array}{c}
-x_{2} \\
x_{1}
\end{array}\right), \quad A(r)=r^{-1} \int_{0}^{r} B(s) s d s .
$$

If $B \in L_{\text {loc }}^{q}\left(\mathbb{R}^{2}, \mathbb{R}\right)$ it is easy to see, using Hölder's inequality, that if $q \in(1,2]$ then $\mathbf{A} \in L_{\text {loc }}^{p}\left(\mathbb{R}^{2} ; \mathbb{R}^{2}\right)$, for some $p \in(2, \infty)$, and that $\mathbf{A} \in L_{\mathrm{loc}}^{\infty}\left(\mathbb{R}^{2} ; \mathbb{R}^{2}\right)$ whenever $q \in(2, \infty]$.

The proof of Theorem 3, given in Subsection 6.3, follows the ideas of [19] consisting in Agmon-type estimates with localizations in space and in the angular momentum variable. Of course, we have to adapt the method of [19] since our Hamiltonian is not bounded form below.

In Subsection 6.1 we transform the operator $H$ to polar coordinates and we decompose it in the angular momentum variable $m_{j}$. The analyticity condition (A4) on $V$ permits us to obtain exponential decay in $\left|m_{j}\right|$ of eigenfunctions of $H$ with eigenvalues $E \in \sigma_{\mathrm{d}}(H)$; see Lemma 5 in Subsection 6.2. In order to obtain the Agmon estimates, in Subsection 6.3, we square the transformed free Dirac operator $K_{0}^{(2)}$; see (39) for its definition. The Gaussian decay is essentially due to a positive term in $\left(K_{0}^{(2)}\right)^{2}$ that goes like $r^{2}$. This term is in competition with a term that behaves like $m_{j}$ when $m_{j} \geq 0$. The Gaussian weights in the Agmon estimates are localized in the region where $m_{j} \lesssim r^{2}$. The complementary region, on the other hand, is controlled by the exponential decay in $\left|m_{j}\right|$. 
6.1. Unitary transform. In the following we derive an equivalent representation of $H$. We denote by $U$ the unitary map that represents $H$ in polar coordinates; see e.g. [30], Section 7.3.3.

$$
\begin{aligned}
U H U^{*} & =H^{(1)}=K_{0}^{(1)}+v(r, \theta), \\
K_{0}^{(1)} & \stackrel{\text { def }}{=} S_{\theta}\left\{-\mathrm{i} \partial_{r}+i r^{-1} \sigma_{3} J_{3}-\mathrm{i} \sigma_{3} A(r)\right\},
\end{aligned}
$$

acting on $\mathscr{H}^{(1)} \stackrel{\text { def }}{=} L^{2}\left(\mathbb{R}^{+}\right) \otimes L^{2}\left(T ; \mathbb{C}^{2}\right)^{2}$, where

$$
J_{3} \stackrel{\text { def }}{=}-\mathrm{i} \partial_{\theta}+1 / 2 \sigma_{3}, \quad S_{\theta} \stackrel{\text { def }}{=}\left(\begin{array}{cc}
0 & e^{-\mathrm{i} \theta} \\
e^{\mathrm{i} \theta} & 0
\end{array}\right) .
$$

Next we identify $L^{2}\left(T ; \mathbb{C}^{2}\right)$ with $\ell^{2}(\mathbb{Z})^{2}$ by means of the transformation

$$
\mathcal{F}: L^{2}\left(T ; \mathbb{C}^{2}\right) \longrightarrow \ell^{2}(\mathbb{Z})^{2}
$$

given by

$$
\mathcal{F}[f](j) \stackrel{\text { def }}{=} \frac{1}{\sqrt{2 \pi}} \int_{0}^{2 \pi} M_{\theta} e^{-\mathrm{i} m_{j} \theta} f(\theta) d \theta,
$$

for $f \in L^{2}(T ; d \theta)^{2}$, where $m_{j}=(2 j+1) / 2, j \in \mathbb{Z}$, and

$$
M_{\theta} \stackrel{\text { def }}{=}\left(\begin{array}{cc}
e^{\mathrm{i} \theta / 2} & 0 \\
0 & \mathrm{i} e^{-\mathrm{i} \theta / 2}
\end{array}\right) \text {. }
$$

Under these transformations we find the decomposition

$$
L^{2}\left(\mathbb{R}^{2} ; \mathbb{C}^{2}\right) \cong \mathscr{H}^{(2)} \stackrel{\text { def }}{=} \bigoplus_{j \in \mathbb{Z}} L^{2}\left(\mathbb{R}^{+} ; d r\right)^{2}
$$

and the corresponding operator

$$
H \cong H^{(2)}=K_{0}^{(2)}+W,
$$

which is essentially self-adjoint on $\mathscr{D}^{(2)} \stackrel{\text { def }}{=} \mathcal{F} U C_{0}^{\infty}\left(\mathbb{R}^{2} ; \mathbb{C}^{2}\right)$. For $h \in D^{(2)}, K_{0}^{(2)}=$ $\mathscr{F} U D_{\mathrm{A}} U^{*} \mathcal{F}^{*}$ acts as

$$
\left(K_{0}^{(2)} h\right)(r, j)=\left(-\mathrm{i} \sigma_{2} \partial_{r}+\sigma_{1}\left(-m_{j} r^{-1}+A(r)\right)\right) h(r, j),
$$

where we used the fact that $\mathcal{F} S_{\theta} \mathscr{F}^{*}=\sigma_{2}$, that $\mathscr{F} S_{\theta} \sigma_{3} \mathscr{F}^{*}=\mathrm{i} \sigma_{1}$, and that $\mathscr{F} J_{3} \mathscr{F}^{*}$ is the multiplication operator by $m_{j}$. The electric potential $W=\mathscr{F}_{\mathcal{F}} v \mathcal{F}^{*}$ acts as

$$
(W h)(r, l) \stackrel{\text { def }}{=} \sum_{j \in \mathbb{Z}} \hat{v}(r, l-j) h(r, j),
$$


where

$$
\hat{v}(r, n)=\frac{1}{2 \pi} \int_{0}^{2 \pi} e^{-\mathrm{i} n \theta} v(r, \theta) d \theta, \quad n \in \mathbb{Z} .
$$

Two other quantities play an important role in our analysis, namely $W_{1} \stackrel{\text { def }}{=} \mathcal{F}_{r} v \mathcal{F}^{*}$ given by

$$
\left(W_{1} h\right)(r, l) \stackrel{\text { def }}{=} \sum_{j \in \mathbb{Z}} \partial_{r} \hat{v}(r, l-j) h(r, j),
$$

and $W_{2} \stackrel{\text { def }}{=} \mathscr{F} \partial_{\theta} v \mathscr{F}^{*}$ that acts as

$$
\left(W_{2} h\right)(r, l) \stackrel{\text { def }}{=} \sum_{j \in \mathbb{Z}} \mathrm{i}(j-l) \hat{v}(r, l-j) h(r, j) .
$$

6.2. Rotation-analyticity. For $f \in \mathscr{H}^{(1)}$ and $a \in \mathbb{R}$ we set

$$
\left(U_{a} f\right)(r, \theta) \stackrel{\text { def }}{=}\left(e^{\mathrm{i} J_{3} a} f\right)(r, \theta)=e^{\mathrm{i} \sigma_{3} a / 2} f(r, \theta+a) .
$$

We call a vector $f \in \mathscr{H}^{(1)}$ rotation-analytic, if and only if the series

$$
\sum_{n \in \mathbb{N}} \frac{\left\|J_{3}^{n} f\right\|}{n !} \rho^{n}, \quad \rho>0,
$$

has an infinite radius of convergence. We start by presenting a lemma that gives us some a priori decay of some eigenfunctions of $H^{(2)}$ in the angular momentum variable.

Lemma 5. Assume that (A1)-(A4) hold. Let $\Psi \in \mathscr{H}^{(2)}$ be an eigenfunction of $H^{(2)}$ to the eigenvalue $E \in \sigma_{d}\left(H^{(2)}\right)$. Then, for every $\gamma>0$, we have

$$
\sum_{j \in \mathbb{Z}} \int_{0}^{\infty} e^{2 \gamma\left|m_{j}\right|}|\Psi(r, j)|^{2} d r<\infty .
$$

Proof. The proof is analog to the one given in [19], Section 3. We sketch it here for the reader's convenience. Due to Lemma 14 (in Appendix B) $\left\{H^{(1)}(z)\right\}_{z \in \mathbb{C}}$ defined on $\mathscr{D}\left(K_{0}^{(1)}\right)$ through

$$
H^{(1)}(z)=K_{0}^{(1)}+\tilde{v}_{z},
$$

is an analytic family of type (A); see [23]. Note that when $a \in \mathbb{R}$ the identity $H^{(1)}(a)=U_{a} H^{(1)} U_{a}^{*}$ holds. Moreover, by Lemma 13 (in Appendix B) we have that

$$
\tilde{v}_{z}\left(K_{0}^{(1)}-\mathrm{i}\right)^{-1}
$$


is a compact operator in $\mathscr{H}^{(1)}$ for any $z \in \mathbb{C}$. Therefore, $\sigma_{\text {ess }}\left(H^{(1)}(z)\right)=\sigma_{\text {ess }}\left(K_{0}^{(1)}\right)$ by Weyl's theorem. Arguing with analytic perturbation theory and using the fact that the spectrum of $H^{(1)}(a)$ and $H^{(1)}$ is the same for $a$ real (see e.g. the proof of Theorem XIII.36 in [23] for a similar argument) we find that $E \in \sigma_{\mathrm{d}}\left(H^{(1)}\right)$ of multiplicity $N \in \mathbb{N}$ is also an eigenvalue of $H^{(1)}(z)$ of the same multiplicity.

Let $P_{z}$ be the $N$-dimensional $E$-eigenprojection of $H^{(1)}(z)$. Since rotationanalytic vectors are dense in $\mathscr{H}^{(1)}$ (see e.g. [20]) we find some rotation-analytic vectors $f_{1}, \ldots, f_{N}$ such that $\operatorname{Ran} P_{0}=\operatorname{Span}\left\{P_{0} f_{1}, \ldots, P_{0} f_{N}\right\}$. Observing that, for $a \in \mathbb{R}$ and $j \in\{1, \ldots, N\}$,

$$
U_{a} P_{0} f_{j}=P_{a} U_{a} f_{j},
$$

we find an analytic continuation of $a \mapsto U_{a} P_{0} f_{j} \in \mathscr{H}^{(1)}$ to the complex plane. In particular, $e^{\mathrm{i} J_{3} z} P_{0} f_{j}$ belongs to $\mathscr{H}^{(1)}$ for any $z \in \mathbb{C}$. Let $\Psi_{1} \in \operatorname{Ran} P_{0}$ be such that $\mathscr{F} \Psi_{1}=\Psi$. By the discussion above we get that

$$
\mathscr{F} e^{J_{3} \gamma} \Psi_{1} \in \mathscr{H}^{(2)}, \quad \gamma \in \mathbb{R} .
$$

This ends the proof since $\left(\mathcal{F} e^{J_{3} \gamma} \Psi_{1}\right)(r, j)=e^{m_{j} \gamma} \Psi(r, j)$ and

$$
\begin{aligned}
& \sum_{j \in \mathbb{Z}} \int_{0}^{\infty} e^{2 \gamma\left|m_{j}\right|}|\Psi(r, j)|^{2} d r \\
& \quad \leq \sum_{j \in \mathbb{Z}} \int_{0}^{\infty} e^{-2 \gamma m_{j}}|\Psi(r, j)|^{2} d r+\sum_{j \in \mathbb{Z}} \int_{0}^{\infty} e^{2 \gamma m_{j}}|\Psi(r, j)|^{2} d r<\infty .
\end{aligned}
$$

6.3. Agmon-type Estimate. In this section we deduce the Agmon estimates needed in the proof of Theorem 3. They were obtained in [19] for the case of a magnetic Schrödinger operator. These estimates uses heavily the fact that the Schrödinger operator is bounded from below. As we commented before we will obtain these estimates for the square of the Dirac operator $K_{0}^{(2)}$.

Fix a number $\tilde{B}>B_{0}$ and note that, due to (A2), there exists $R_{0}>0$ so large that the estimate (47) is fulfilled and moreover

$$
\left\|1_{\left\{r>R_{0}\right\}} B\right\|<\tilde{B}, \quad r>R_{0} .
$$

We set, for $0<q_{2}<q_{1}<1$,

and

$$
r(j) \stackrel{\text { def }}{=} \begin{cases}\sqrt{\frac{4 \tilde{B}}{\left(q_{1}^{2}-q_{2}^{2}\right) B_{0}^{2}} m_{j}} & m_{j} \geq 0 \\ 0 & m_{j}<0,\end{cases}
$$

$$
\Omega_{q_{1}, q_{2}} \stackrel{\text { def }}{=}\left\{(r, j) \in \mathbb{R}^{+} \times \mathbb{Z} \mid r \geq r(j)\right\} .
$$


Moreover, we define

$$
\rho(r, j) \stackrel{\text { def }}{=} \begin{cases}q_{2} B_{0} / 4\left(r^{2}-r(j)^{2}\right) & m_{j} \geq 0, r \geq r(j), \\ q_{2} B_{0} r^{2} / 4 & m_{j}<0, \\ 0 & m_{j} \geq 0, r<r(j) .\end{cases}
$$

Eventually we will choose $q_{2}$ to be sufficiently close to 1 . A direct calculation shows that

$$
\left|\rho\left(r, j_{1}\right)-\rho\left(r, j_{2}\right)\right| \leq \frac{q_{2} \tilde{B}}{\left(q_{1}^{2}-q_{2}^{2}\right) B_{0}}\left|j_{1}-j_{2}\right| .
$$

Let $\rho_{\varepsilon} \stackrel{\text { def }}{=} \rho(1+\varepsilon \rho)^{-1}$. It is easy to see that

$$
\left|\rho_{\varepsilon}\left(r, j_{1}\right)-\rho_{\varepsilon}\left(r, j_{2}\right)\right| \leq \frac{q_{2} \tilde{B}}{\left(q_{1}^{2}-q_{2}^{2}\right) B_{0}}\left|j_{1}-j_{2}\right| .
$$

Finally, for $R>R_{0}$, we fix a smooth function $f_{R}$ in $r$ with bounded derivatives in $\mathbb{R}^{+} \times \mathbb{Z}$ satisfying

$$
f_{R}(r, j)= \begin{cases}1 & r \geq 2 R \text { and }(r, j) \in \Omega_{q_{1}, q_{2}}, \\ 0 & r \leq R \text { or }(r, j) \notin \Omega_{q_{1}, \mu q_{2}},\end{cases}
$$

where $\mu \in(0,1)$ is a fixed number that will be chosen sufficiently close to 1 . Note that $\Omega_{q_{1}, q_{2}} \subset \Omega_{q_{1}, \mu q_{2}}$.

Let $\Psi$ be the eigenfunction from Theorem 3 and $\hat{\Psi} \stackrel{\text { def }}{=} \mathcal{F} U \Psi$ be a normalized eigenfunction of $H^{(2)}$ with corresponding energy $E \in \sigma_{d}\left(H^{(2)}\right)$. We set, for $R>R_{0}$ and $\delta \in(0, \mu)$,

$$
g \stackrel{\text { def }}{=} e^{\delta \rho_{\varepsilon}} f_{R} \widehat{\Psi}
$$

Observe that $\delta$ can be chosen arbitrarily close to 1 .

Lemma 6. We find constants $R_{1}>R_{0}$ and $c>0$ such that, for any $\delta \in(-1,1), \rho>$ $R_{1}$, and $j \in\{0,1,2\}$,

$$
\sup _{\varepsilon>0}\left\|\theta_{\rho} e^{\delta \rho_{\varepsilon}} W_{j} e^{-\delta \rho_{\varepsilon}} \theta_{\rho}\right\|<c
$$

where $W_{0} \stackrel{\text { def }}{=} W$ and $\theta_{\rho} \stackrel{\text { def }}{=} \mathbb{1}_{\{r>\rho\}}$. In particular, the commutator

$$
\left[K_{0}^{(2)}, W_{0}\right]=-\mathrm{i} \sigma_{2} W_{1}+\frac{\mathrm{i} \sigma_{1}}{r} W_{2}
$$

satisfies the estimate

$$
\sup _{\varepsilon>0}\left\|e^{\delta \rho_{\varepsilon}} f_{R}\left[K_{0}^{(2)}, W_{0}\right] e^{-\delta \rho_{\varepsilon}}\right\|<2 c, \quad R>R_{1} .
$$


Proof. We show (47) only for $j=2$, for the other cases follow analogously. For any $m \in \mathbb{Z}$ and $r>0$ we define the analytic function $\mathbb{C} \ni z \mapsto h_{m}(r, z) \stackrel{\text { def }}{=} e^{-\mathrm{i} m z} \tilde{v}_{z}(r, 0)$. Using (41) and the decay and analyticity assumptions on $v$ (A5) we find for any $r>\rho$ (sufficiently large), $m \in \mathbb{Z}$, and $\gamma \in \mathbb{R}$ that there is a constant $C>0$ such that

$$
\begin{aligned}
|\widehat{v}(r, m)| & =\frac{1}{2 \pi}\left|\int_{0}^{2 \pi} h_{m}(r, \theta) d \theta\right|=\frac{1}{2 \pi}\left|\int_{0}^{2 \pi} h_{m}(r, \theta-\mathrm{i} \gamma) d \theta\right| \\
& \leq \frac{e^{-m \gamma}}{2 \pi} \int_{0}^{2 \pi} u_{2|\gamma|}(r, \theta) d \theta \leq\left\|\theta_{\rho} u_{2|\gamma|}\right\|_{\infty} e^{-m \gamma} \leq C e^{-m \gamma} .
\end{aligned}
$$

Here we also used Cauchy's integral theorem and the fact that $h_{m}(r, z)$ is $2 \pi$-periodic with respect to $\operatorname{Re}(z)$. In particular, replacing $\gamma$ by $-\gamma$ in the above estimate we see that, for $\gamma>0$ and $m \in \mathbb{Z}$, the bound $\theta_{\rho}|\widehat{v}(r, m)| \leq C e^{-|m| \gamma}$ holds. Therefore, using (45), (43), and Young's inequality for $\ell^{2}\left(\mathbb{Z}^{2} ; \mathbb{C}^{2}\right)$ in combination with the Cauchy-Schwarz inequality for $L^{2}((0, \infty) ; \mathbb{C})$, we get, for $\gamma$ sufficiently large and every $f \in \mathscr{H}^{(2)}$, that $\left|\left\langle f, \theta_{\rho} e^{\delta \rho_{\varepsilon}} W_{2} e^{-\delta \rho_{\varepsilon}} \theta_{\rho} f\right\rangle\right|$ is bounded by

$$
\int_{0}^{\infty} \sum_{l \in \mathbb{Z}}|f(r, l)| \sum_{j \in \mathbb{Z}}\left|\theta_{\rho} \hat{v}(r, l-j)\right||l-j| e^{\delta \tau|l-j|}|f(r, j)| d r \leq \widetilde{C}\|f\|^{2},
$$

for some constant $\widetilde{C}>0$, where $\tau \stackrel{\text { def }}{=} \frac{q_{2} \tilde{B}}{\left(q_{1}^{2}-q_{2}^{2}\right) B_{0}}$.

Equation (49) follows from (47) and (48). Equation (48) is a consequence of

$$
\begin{aligned}
{\left[K_{0}^{(2)}, W\right] } & =\mathcal{F}\left[K_{0}^{(1)}, v\right] \mathcal{F}^{*} \\
& =\mathscr{F}\left(-\mathrm{i} S_{\theta} \partial_{r} v+\frac{S_{\theta} \sigma_{3}}{r} \partial_{\theta} v\right) \mathcal{F}^{*},
\end{aligned}
$$

and the fact that $\mathscr{F} S_{\theta} \mathscr{F}^{*}=\sigma_{2}$ and $\mathscr{F} S_{\theta} \sigma_{3} \mathcal{F}^{*}=\mathrm{i} \sigma_{1}$.

Before continuing let us state a simple technical result.

Lemma 7. For any $\gamma \in \mathbb{R}$ we have that $e^{\gamma \rho_{\varepsilon}} f_{R} \hat{\Psi} \in \mathscr{D}\left(K_{0}^{(2)}\right)$.

Proof. Let $\lambda>0$ and $\eta \in \mathscr{F} U C_{0}^{\infty}\left(\mathbb{R}^{2} ; \mathbb{C}^{2}\right)$. First observe that a simple computation shows that

$$
\left(\partial_{r} e^{\gamma \rho_{\varepsilon}} f_{R}\right) e^{-\lambda r}
$$

extends to a bounded operator on $\mathscr{H}^{(2)}$. In addition, $e^{\lambda r} \hat{\Psi} \in \mathscr{H}^{(2)}$ by Theorem 2. Therefore, we get by explicit calculation on $\mathscr{F} U C_{0}^{\infty}\left(\mathbb{R}^{2} ; \mathbb{C}^{2}\right)$, that

$$
\begin{aligned}
\left\langle K_{0}^{(2)} \eta, e^{\gamma \rho_{\varepsilon}} f_{R} \hat{\Psi}\right\rangle & =\left\langle f_{R} e^{\gamma \rho_{\varepsilon}} K_{0}^{(2)} \eta, \widehat{\Psi}\right\rangle \\
& =\left\langle K_{0}^{(2)} f_{R} e^{\gamma \rho_{\varepsilon}} \eta, \widehat{\Psi}\right\rangle+\left\langle i \sigma_{2}\left(\partial_{r} e^{\gamma \rho_{\varepsilon}} f_{R}\right) \eta, \widehat{\Psi}\right\rangle \\
& =\left\langle\eta, e^{\gamma \rho_{\varepsilon}} f_{R} K_{0}^{(2)} \hat{\Psi}\right\rangle-\mathrm{i}\left\langle\eta, \sigma_{2}\left(\partial_{r} e^{\gamma \rho_{\varepsilon}} f_{R}\right) e^{-\lambda r}\left(e^{\lambda r} \hat{\Psi}\right)\right\rangle .
\end{aligned}
$$


Since $\eta$ can be chosen arbitrarily from the domain of essential self-adjointness of $K_{0}^{(2)}$ we get the desired result.

An important role in our analysis is played by the quantity

$$
Q \stackrel{\text { def }}{=} \operatorname{Re}\left\langle K_{0}^{(2)} e^{\delta \rho_{\varepsilon}} g, K_{0}^{(2)} e^{-\delta \rho_{\varepsilon}} g\right\rangle,
$$

which is well defined due to Lemma 7. Before we show Theorem 3 we state two preparatory lemmata whose proofs are given in the next subsection.

Lemma 8. There are $R, \varepsilon$-independent constants $C_{1}, C_{2}>0$ such that, for $R>R_{1}$ sufficiently large,

$$
Q \geq\left(C_{1} R^{2}-C_{2}\right)\|g\|^{2} .
$$

Lemma 9. There is an $R, \varepsilon$-independent constant $C_{3}$ and an $\varepsilon$-independent constant $C(R)$ such that, for $R>R_{1}$ sufficiently large,

$$
Q \leq C_{3}\|g\|^{2}+C(R)\|g\| .
$$

Proof of Theorem 3. Fix $\delta, q_{1}, q_{2} \in(0,1)$. Combining Lemma 9 and 8 we find, for $R>R_{1}$ sufficiently large,

$$
\|g\| \leq\left(C_{1} R^{2}-C_{2}-C_{3}\right)^{-1} C(R)
$$

Since the right hand side of (54) is independent of $\varepsilon$ we obtain, by the monotone convergence theorem,

$$
\left\|e^{\delta \rho} \hat{\Psi}\right\|^{2}=\lim _{\varepsilon \rightarrow 0}\left\|e^{\delta \rho_{\varepsilon}} \hat{\Psi}\right\|^{2} \leq\left(\sup _{\varepsilon>0}\|g\|+\left\|e^{\delta \rho}\left(1-f_{R}\right)\right\|\right)^{2}<\infty .
$$

For $M>1$ define

$$
\widetilde{\Omega}_{q_{1}, q_{2}, M}=\left\{(r, j) \in \mathbb{R}^{+} \times \mathbb{Z} \mid r^{2} \geq \operatorname{Mr}(j)^{2}\right\} .
$$

We have that $\widetilde{\Omega}_{q_{1}, q_{2}, M} \subset \Omega_{q_{1}, q_{2}}$. Thus, for any $(r, j) \in \widetilde{\Omega}_{q_{1}, q_{2}, M}$, we get

$$
\rho(r, j)=\frac{q_{2} B_{0}}{4}\left(r^{2}-r(j)^{2}\right) \geq \frac{q_{2} B_{0}}{4}\left(1-\frac{1}{M}\right) r^{2} .
$$

Therefore, setting $\alpha \stackrel{\text { def }}{=} \delta q_{2}\left(1-M^{-1}\right)$, we obtain

$$
\left\|e^{\alpha B_{0} / 4 r^{2}} \mathbb{1}_{\widetilde{\Omega}_{q_{1}, q_{2}, M}} \hat{\Psi}\right\|<\infty .
$$

If $(r, j) \notin \widetilde{\Omega}_{q_{1}, q_{2}, M}$ then

$$
m_{j} \geq \frac{\left(q_{1}^{2}-q_{2}^{2}\right) B_{0}^{2} r^{2}}{4 M \tilde{B}} \stackrel{\text { def }}{=} \beta r^{2} .
$$


Thus, thanks to Lemma 5 we deduce, for any $\gamma>0$, that

$$
\left\|e^{\beta \gamma r^{2}} \mathbb{1}_{\tilde{\Omega}_{q_{1}, q_{2}, M}^{c}} \hat{\Psi}\right\|<\infty .
$$

Choosing $\gamma=\alpha / \beta \cdot B_{0} / 4$ and combining (56) with (55) we conclude that

$$
\left\|e^{\alpha B_{0} / 4 r^{2}} \hat{\Psi}\right\|<\infty .
$$

The latter holds for $\alpha>0$ arbitrarily close to 1 , since $\delta$ and $q_{2}$ can be chosen arbitrarily close to 1 and $M>1$ can be as large as we want. This proves the theorem.

6.4. Proof of Lemmata 8 and 9. Before we give the proof of Lemmata 8 and 9 we need a preparatory result.

Lemma 10. For $R>R_{1}$ sufficiently large we have that

$$
\left\|K_{0}^{(2)} g\right\|_{\mathscr{H}^{(2)}}^{2} \geq \mu^{2} q_{2}^{2} B_{0}^{2}\|r g\|^{2} / 4-\left\|r^{-1} g\right\|^{2} / 4-\tilde{B}\|g\|^{2} .
$$

Proof. Let us write $g=\left(g^{+}, g^{-}\right)^{\mathrm{T}}$ and $g_{j}^{ \pm} \stackrel{\text { def }}{=} g^{ \pm}(\cdot, j)$. By Equation (39) we have

$$
\begin{aligned}
& \left\|K_{0}^{(2)} g\right\|_{\mathcal{H}^{(2)}}^{2} \\
& \quad=\sum_{j \in \mathbb{Z}}\left(\left\|\left(\partial_{r}-m_{j} r^{-1}+A(r)\right) g_{j}^{+}\right\|^{2}+\left\|\left(-\partial_{r}-m_{j} r^{-1}+A(r)\right) g_{j}^{-}\right\|^{2}\right) .
\end{aligned}
$$

Furthermore, dropping the term $-\partial_{r}^{2}$, we get

$$
\begin{aligned}
& \left\|\left( \pm \partial_{r}-m_{j} r^{-1}+A(r)\right) g_{j}^{ \pm}\right\|^{2} \\
& \left.\quad \geq\left\langle g_{j}^{ \pm},\left(\left(m_{j}^{2} \mp m_{j}\right) r^{-2}+A(r)^{2}\right) g_{j}^{ \pm}\right\rangle+\left\langle g_{j}^{ \pm}, \mp \partial_{r} A(r)-2 m_{j} r^{-1} A(r)\right) g_{j}^{ \pm}\right\rangle .
\end{aligned}
$$

Observe that (A2) implies that

$$
\frac{1}{r^{2}} \int_{0}^{r} b(s) s d s=\mathrm{o}(1), \quad \text { as } r \rightarrow \infty .
$$

This can be seen by splitting the integral above in the regions where $b(s) s$ is integrable and the one where $b$ decays in the $L^{\infty}$ - norm. Hence, given $q_{3} \in\left(q_{1}, 1\right)$ we find, using (57), a constant $R_{2}>R_{1}$ such that, for all $r>R_{2}$,

$$
\begin{aligned}
& B(r) \geq q_{3} B_{0}, \quad A(r) \geq q_{1} B_{0} r / 2, \\
& \left|\partial_{r} A(r)\right| \leq \tilde{B}, \quad A(r) \leq \tilde{B} r / 2 .
\end{aligned}
$$

Therefore, for all $r>R>R_{2}$, we get

$$
\begin{aligned}
& \left\|\left( \pm \partial_{r}-m_{j} r^{-1}+A(r)\right) g_{j}^{ \pm}\right\|^{2} \\
& \quad \geq\left\langle g_{j}^{ \pm},\left(-r^{-2} / 4+q_{1}^{2} B_{0}^{2} r^{2} / 4-2 m_{j} r^{-1} A(r)-\tilde{B}\right) g_{j}^{ \pm}\right\rangle,
\end{aligned}
$$


where we also use that $\left(m_{j}^{2} \pm m_{j}\right) \geq-1 / 4$.

Assume that $m_{j}<0$. Since $q_{1}>q_{2}$ and $A(r)>0$, for $r>R_{2}$, we find that

$$
\left\|\left( \pm \partial_{r}-m_{j} r^{-1}+A(r)\right) g_{j}^{ \pm}\right\|^{2} \geq\left\langle g_{j}^{ \pm} \mid\left(q_{2}^{2} B_{0}^{2} r^{2} / 4-r^{-2} / 4-\tilde{B}\right) g_{j}^{ \pm}\right\rangle .
$$

Assume now that $m_{j} \geq 0$. Recall that $A(r) \leq \tilde{B} r / 2$, for $r>R_{2}$. Using that $m_{j} \leq r^{2}\left(q_{1}^{2}-\mu^{2} q_{2}^{2}\right) B_{0}^{2} /(4 \tilde{B})$ on supp $g \subset \Omega_{q_{1}, \mu q_{2}}$ we get

$$
\begin{aligned}
& \left\|\left( \pm \partial_{r}-m_{j} r^{-1}+A(r)\right) g_{j}^{ \pm}\right\|^{2} \\
& \quad \geq\left\langle g_{j}^{ \pm} \mid\left(q_{1}^{2} B_{0}^{2} r^{2} / 4-r^{-2} / 4-m_{j} \tilde{B}-\tilde{B}\right) g_{j}^{ \pm}\right\rangle \\
& \quad \geq\left\langle g_{j}^{ \pm} \mid\left(\mu^{2} q_{2}^{2} B_{0}^{2} r^{2} / 4-r^{-2} / 4-\tilde{B}\right) g_{j}^{ \pm}\right\rangle .
\end{aligned}
$$

This finishes the proof.

Proof of Lemma 8. Notice that

$$
e^{ \pm \delta \rho_{\varepsilon}} K_{0}^{(2)} e^{\mp \delta \rho_{\varepsilon}}=K_{0}^{(2)}+Z^{ \pm \rho_{\varepsilon}}, \quad Z^{ \pm \rho_{\varepsilon}} \stackrel{\text { def }}{=} \pm \mathrm{i} \delta \partial_{r} \rho_{\varepsilon} \sigma_{2} .
$$

Thus, we have

$$
\begin{aligned}
Q & =\operatorname{Re}\left\langle\left(K_{0}^{(2)}+Z^{-\rho_{\varepsilon}}\right) g \mid\left(K_{0}^{(2)}+Z^{\rho_{\varepsilon}}\right) g\right\rangle \\
& =\left\|K_{0}^{(2)} g\right\|^{2}-\delta^{2}\left\|\partial_{r} \rho_{\varepsilon} g\right\|^{2} .
\end{aligned}
$$

Since $\left|\partial_{r} \rho_{\varepsilon}\right| \leq\left|\partial_{r} \rho\right| \leq q_{2} B_{0} r / 2$ we find

$$
Q \geq\left\|K_{0}^{(2)} g\right\|^{2}-(1 / 4) \delta^{2} q_{2}^{2} B_{0}^{2}\|r g\|^{2} .
$$

Combining this with Lemma 10 and that supp $g \subset\{(r, j) \mid r \geq R\}$ we obtain (recall that $0<\delta<\mu<1)$

$$
Q \geq\left(\left(\mu^{2}-\delta^{2}\right) q_{2}^{2} B_{0}^{2} R^{2} / 4-R^{-2} / 4-\tilde{B}\right)\|g\|^{2} .
$$

This concludes the proof.

Proof of Lemma 9. We clearly have

$$
Q \leq\left|\left\langle K_{0}^{(2)} e^{\delta \rho_{\varepsilon}} g, f_{R}(E-W) \hat{\Psi}\right\rangle\right|+\left|\left\langle K_{0}^{(2)} e^{\delta \rho_{\varepsilon}} g, \sigma_{2}\left(\partial_{r} f_{R}\right) \hat{\Psi}\right\rangle\right| .
$$

We analyze each of the above terms separately. Using that $\left(K_{0}^{(2)}+W\right) \hat{\Psi}=E \hat{\Psi}$ and noting that $W f_{R}$ extends trivially to a bounded operator (for $R>R_{1}$ large enough), we have, for any $\eta \in \mathcal{F} U C_{0}^{\infty}\left(\mathbb{R}^{2} ; \mathbb{C}^{2}\right)$,

$$
\begin{aligned}
\left\langle K_{0}^{(2)}\right. & \left.\eta, f_{R}(E-W) \hat{\Psi}\right\rangle \\
& =\left\langle(E-W) f_{R} K_{0}^{(2)} \eta, \widehat{\Psi}\right\rangle \\
& =\left\langle K_{0}^{(2)} f_{R}(E-W) \eta, \widehat{\Psi}\right\rangle+\left\langle\left[(E-W) f_{R}, K_{0}^{(2)}\right] \eta, \widehat{\Psi}\right\rangle \\
& =\left\langle\eta,(E-W)^{2} f_{R} \hat{\Psi}\right\rangle+\left\langle\eta,\left[W, K_{0}^{(2)}\right] f_{R} \hat{\Psi}\right\rangle+\left\langle\eta, \mathrm{i} \sigma_{2}\left(\partial_{r} f_{R}\right)(W-E) \hat{\Psi}\right\rangle .
\end{aligned}
$$


This identity extends to any $\eta \in \mathscr{D}\left(K_{0}^{(2)}\right)$, in particular, we may choose $\eta=e^{\delta \rho_{\varepsilon}} g$ (see Lemma 7). Thus, using Lemma 6, we find a constant $C>0$, independent of $R$ and $\varepsilon$, such that

$$
\begin{aligned}
& \left|\left\langle K_{0}^{(2)} e^{\delta \rho_{\varepsilon}} g, f_{R}(E-W) \hat{\Psi}\right\rangle\right| \\
& \quad \leq\|g\|\left\|e^{\delta \rho_{\varepsilon}}\left[(E-W)^{2} f_{R}+\left[W, K_{0}^{(2)}\right] f_{R}+\mathrm{i} \sigma_{2}\left(\partial_{r} f_{R}\right)(W-E)\right] \widehat{\Psi}\right\| \\
& \quad \leq C\|g\|\left\|e^{\delta \rho_{\varepsilon}} \hat{\Psi}\right\| \leq C\|g\|\left(\|g\|+\left\|e^{\delta \rho}\left(1-f_{R}\right)\right\|\right) .
\end{aligned}
$$

We now treat the second term in (59). We define the operators $\Upsilon$ and $L$ acting, for any $h \in \mathscr{H}^{(2)}$ and $(r, j) \in \mathbb{R}^{+} \times \mathbb{Z}$, as

$$
\begin{aligned}
& (\Upsilon h)(r, j)=e^{-\left|m_{j}\right|} h(r, j), \\
& (L h)(r, j)=\left(2 \sigma_{1} \sigma_{2}\left(m_{j} r^{-1}+A(r)\right)\left(\partial_{r} f_{R} \Upsilon h\right)(r, j) .\right.
\end{aligned}
$$

Clearly, since $A(r)$ is bounded on the support of $\partial_{r} f_{R}-$ for $R>R_{1}$ large enough; see (58) $-L$ is an anti-symmetric bounded operator on $\mathscr{H}^{(2)}$. With these definitions we have, using again the eigenvalue equation, that for any $\eta \in \mathscr{F} U C_{0}^{\infty}\left(\mathbb{R}^{2} ; \mathbb{C}^{2}\right)$

$$
\begin{aligned}
& \left\langle K_{0}^{(2)} \eta, \sigma_{2}\left(\partial_{r} f_{R}\right) \hat{\Psi}\right\rangle \\
& =\left\langle K_{0}^{(2)} \sigma_{2}\left(\partial_{r} f_{R}\right) \eta, \hat{\Psi}\right\rangle+\left\langle\eta, \mathbb{1}_{\text {supp } \partial_{r} f_{R}}\left(\mathrm{i} \partial_{r}^{2} f_{R} \widehat{\Psi}-L \Upsilon^{-1} \widehat{\Psi}\right)\right\rangle \\
& =\left\langle\eta, \mathbb{1}_{\text {supp } \partial_{r} f_{R}}\left(\sigma_{2}\left(\partial_{r} f_{R}\right)(E-W) \hat{\Psi}+\mathrm{i} \partial_{r}^{2} f_{R} \widehat{\Psi}-L \Upsilon^{-1} \hat{\Psi}\right)\right\rangle .
\end{aligned}
$$

Note that $\Upsilon^{-1} \hat{\Psi} \in \mathscr{H}^{(2)}$ by Lemma 5. Next, we extend this identity to $\eta \in \mathscr{D}\left(K_{0}^{(2)}\right)$ and replace $\eta$ by $e^{\delta \rho_{\varepsilon}} g$. Using that $e^{\delta \rho_{\varepsilon}} \mathbb{1}_{\text {supp } \partial_{r} f_{R}}$ is bounded uniformly in $\varepsilon>0$, we find $\varepsilon$-independent constants $C(R), C^{\prime}(R)>0$ such that

$$
\begin{aligned}
& \left|\left\langle K_{0}^{(2)} e^{\delta \rho_{\varepsilon}} g, \sigma_{2}\left(\partial_{r} f_{R}\right) \hat{\Psi}\right\rangle\right| \\
& \quad \leq C^{\prime}(R)\|g\|\left\|e^{\delta \rho_{1}} \mathbb{1}_{\text {supp } \partial_{r} f_{R}}\right\|\left(\left\|\Upsilon^{-1} \widehat{\Psi}\right\|+\left\|\mathbb{1}_{\text {supp } \partial_{r} f_{R}} W \widehat{\Psi}\right\|\right) \\
& \quad \leq C(R)\|g\|,
\end{aligned}
$$

where in the last inequality we use again Lemma 5. Therefore, we obtain from (59) and the above bounds that

$$
Q \leq\|g\|\left(C\|g\|+C\left\|e^{\delta \rho}\left(1-f_{R}\right)\right\|+C(R)\right),
$$

which concludes the proof. 


\section{A. Bounds for the Green function of $D_{\mathrm{A}_{0}}$}

Let

$$
\begin{aligned}
& \theta\left(\mathbf{x}-\mathbf{x}^{\prime}\right) \stackrel{\text { def }}{=} \frac{B_{0}\left|\mathbf{x}-\mathbf{x}^{\prime}\right|^{2}}{4}, \\
& \eta\left(\mathbf{x}, \mathbf{x}^{\prime}\right) \stackrel{\text { def }}{=}-\frac{B_{0}}{2}\left(x_{1} x_{2}^{\prime}-x_{2} x_{1}^{\prime}\right) .
\end{aligned}
$$

Lemma 11. Let $z \in \mathbb{R} \backslash \sigma\left(D_{\mathbf{A}_{0}}\right)$ and let $G_{0}\left(\mathbf{x}, \mathbf{x}^{\prime}, z\right)$, $\mathbf{x}, \mathbf{x}^{\prime} \in \mathbb{R}^{2}$, be a representation of the Green kernel of $\left(D_{\mathbf{A}_{0}}-z\right)^{-1}$ as $2 \times 2$-matrix. Then we have that

$$
\left\|G_{0}\left(\mathbf{x}, \mathbf{x}^{\prime} ; z\right)\right\|_{\mathbb{C}^{2} \otimes \mathbb{C}^{2}} \leq e^{-\theta\left(\mathbf{x}-\mathbf{x}^{\prime}\right)} \omega\left(\mathbf{x}-\mathbf{x}^{\prime} ; z\right),
$$

for some function $\omega(\cdot ; z): \mathbb{R}^{2} \rightarrow \mathbb{R}^{+}$that satisfies

$$
\sup _{\mathbf{x} \in \mathbb{R}^{2}}|\mathbf{x}| e^{-\varepsilon \mid \mathbf{x}} \omega(\mathbf{x} ; z)<\infty, \quad \varepsilon>0 .
$$

Proof. Recall that by Proposition 1 we have for $E \neq 0$ that $\pm E \in \sigma\left(D_{\mathbf{A}_{0}}\right)$ if and only if $E^{2} \in \sigma\left(d d^{*}\right) \backslash\{0\}=\sigma\left(d^{*} d\right) \backslash\{0\}$, where

$$
\begin{aligned}
& d^{*} d=\left(\mathbf{p}-\mathbf{A}_{0}\right)^{2}-B_{0}, \\
& d d^{*}=\left(\mathbf{p}-\mathbf{A}_{0}\right)^{2}+B_{0} .
\end{aligned}
$$

A simple computation using (6) yields, for any $z \in \mathbb{R} \backslash \sigma\left(D_{\mathbf{A}_{0}}\right)$,

$$
\begin{aligned}
\left(D_{\mathbf{A}_{0}}-z\right)^{-1} & =\left(D_{\mathbf{A}_{0}}+z\right)\left(D_{\mathbf{A}_{0}}^{2}-z^{2}\right)^{-1} \\
& =\left(\begin{array}{ll}
z\left(d^{*} d-z^{2}\right)^{-1} & d^{*}\left(d d^{*}-z^{2}\right)^{-1} \\
d\left(d^{*} d-z^{2}\right)^{-1} & z\left(d d^{*}-z^{2}\right)^{-1}
\end{array}\right) .
\end{aligned}
$$

It is well-known that the Green function of $\left(\mathbf{p}-\mathbf{A}_{0}\right)^{2}$ is given by

$$
\begin{aligned}
& {\left[\left(\mathbf{p}-\mathbf{A}_{0}\right)^{2}-\zeta\right]^{-1}\left(\mathbf{x}, \mathbf{x}^{\prime}\right)} \\
& \quad=(4 \pi)^{-1} \Gamma(\alpha) e^{\mathrm{i} \eta\left(\mathbf{x}, \mathbf{x}^{\prime}\right)} e^{-\theta\left(\mathbf{x}-\mathbf{x}^{\prime}\right)} U\left(\alpha, 1,2 \theta\left(\mathbf{x}-\mathbf{x}^{\prime}\right)\right),
\end{aligned}
$$

where $U$ is a confluent hypergeometric function and $\alpha=-1 / 2\left(\zeta / B_{0}-1\right) \notin-\mathbb{N}$; see for instance [6], Lemma 2.2. by

Combining (62), (63), and (64) we obtain that the Green kernel of $D_{\mathbf{A}_{0}}$ is given

$$
G_{0}\left(\mathbf{x}, \mathbf{x}^{\prime} ; z\right)=e^{\mathrm{i} \eta\left(\mathbf{x}, \mathbf{x}^{\prime}\right)-\theta\left(\mathbf{x}-\mathbf{x}^{\prime}\right)}\left(\begin{array}{ll}
\Omega_{11}\left(\mathbf{x}, \mathbf{x}^{\prime} ; z\right) & \Omega_{12}\left(\mathbf{x}, \mathbf{x}^{\prime} ; z\right) \\
\Omega_{12}\left(\mathbf{x}, \mathbf{x}^{\prime} ; z\right) & \Omega_{22}\left(\mathbf{x}, \mathbf{x}^{\prime} ; z\right)
\end{array}\right),
$$


where we define $\alpha_{ \pm}=-1 / 2\left(\left(z^{2} \pm B_{0}\right) / B_{0}-1\right)$ and

$$
\begin{aligned}
& \Omega_{11}\left(\mathbf{x}, \mathbf{x}^{\prime} ; z\right) \stackrel{\text { def }}{=}(4 \pi)^{-1} z \Gamma\left(\alpha_{+}\right) U\left(\alpha_{+}, 1,2 \theta\left(\mathbf{x}-\mathbf{x}^{\prime}\right)\right), \\
& \Omega_{12}\left(\mathbf{x}, \mathbf{x}^{\prime} ; z\right) \\
& \quad \stackrel{\text { def }}{=}(4 \pi)^{-1} B_{0} \Gamma\left(\alpha_{-}+1\right) U\left(\alpha_{-}+1,2,2 \theta\left(\mathbf{x}-\mathbf{x}^{\prime}\right)\right)\left\{\mathrm{i}\left(x_{1}-x_{1}^{\prime}\right)+\left(x_{2}-x_{2}^{\prime}\right)\right\}, \\
& \Omega_{22}\left(\mathbf{x}, \mathbf{x}^{\prime} ; z\right) \stackrel{\text { def }}{=}(4 \pi)^{-1} z \Gamma\left(\alpha_{-}\right) U\left(\alpha_{-}, 1,2 \theta\left(\mathbf{x}-\mathbf{x}^{\prime}\right)\right) .
\end{aligned}
$$

Here we also used that $\frac{d}{d t} U(\alpha, 1, t)=-\alpha_{-} U\left(\alpha_{-}+1,2, t\right)$; see [1], eq. (13.4.22). Since $-\alpha_{ \pm} \notin \mathbb{N}_{0}$, the bounds (60) and (61) follow now from the asymptotic formulae for $U$; see [1], eq. (13.5.2), eq. (13.5.7), and eq. (13.5.9).

\section{B. The family $\left\{H^{(1)}(z)\right\}_{z \in \mathbb{C}}$}

Throughout this section we assume that (A1)-(A4) are satisfied and use that notation introduced in Section 6. Our concern is the family of operators $\left\{H^{(1)}(z)\right\}_{z \in \mathbb{C}}$ defined a priori on the dense subspace $U C_{0}^{\infty}\left(\mathbb{R}^{2}, \mathbb{C}^{2}\right)$ of $\mathscr{H}^{(1)}$ as

$$
H^{(1)}(z) \stackrel{\text { def }}{=} K_{0}^{(1)}+\tilde{v}_{z}, \quad z \in \mathbb{C} .
$$

We first state a technical lemma.

Lemma 12. Let $T$ be a (complex-valued) multiplication operator on $L^{2}\left(\mathbb{R}^{2}, \mathbb{C}^{2}\right)$ with $T \in L_{\text {loc }}^{p}\left(\mathbb{R}^{2}, \mathbb{C}^{2}\right), p \in(2, \infty]$ and $\lim _{n \rightarrow \infty}\left\|\mathbb{1}_{\{|\mathbf{x}|>n\}} T\right\|_{\infty}=0$. Then, $T$ is relative $\sqrt{\mathbf{p}^{2}+1}$-compact.

Proof. For $n \in \mathbb{N}$ write $T=T_{1}+T_{2}$ where $T_{1}$ is supported inside the ball $B_{n}(0) \subset$ $\mathbb{R}^{2}$ and $T_{2}$ on the complement of $B_{n}(0)$. Then $T_{1}$ is relative $\sqrt{\mathbf{p}^{2}+1}$-compact; see [28], Theorem 4.1. Moreover,

$$
\left\|T\left(\mathbf{p}^{2}+1\right)^{-1 / 2}-T_{1}\left(\mathbf{p}^{2}+1\right)^{-1 / 2}\right\| \leq\left\|T_{2}\right\| \longrightarrow 0,
$$

as $n \rightarrow \infty$, from which follows the claim.

Lemma 13. For any $z \in \mathbb{C}$ the operator $\widetilde{v}_{z}\left(K_{0}^{(1)}+\mathrm{i}\right)^{-1}$ is compact in $\mathscr{H}^{(1)}$.

Proof. Let $z \in \mathbb{C}$ and $\tau>0$ with $\tau>|z|$. Due to the inequality $\left|\tilde{v}_{z}\right| \leq u_{\tau}$ on $\mathbb{R}^{+} \times T$ and the fact that $u_{\tau} \in L^{p}\left(\mathbb{R}^{+} \times T, r d r d \theta\right)$ (for some $2<p \leq \infty$ ) we see that $\tilde{v}_{z}$ is well defined on the domain of $K_{0}^{(1)}$. Let $\tilde{u}_{\tau}=U^{*} u_{\tau} U$. It suffices to show that $U^{*} \tilde{v}_{z} U\left(D_{\mathbf{A}}+\mathrm{i}\right)^{-1}$ is compact in $L^{2}\left(\mathbb{R}^{2} ; \mathbb{C}^{2}\right)$. This is, however, a consequence of Lemma 12 and the discussion at the end of the proof of Lemma 1. 
Lemma 14. $\left\{H^{(1)}(z)\right\}_{z \in \mathbb{C}}$ defined in (65) extends to an analytic family of type (A) with domain $\mathscr{D}\left(H^{(1)}(z)\right)=\mathscr{D}\left(K_{0}^{(1)}\right)$.

Proof. Due to Lemma 13 we know that, for any $z \in \mathbb{C}, H^{(1)}(z)$ extends to a closed operator with $\mathscr{D}\left(H^{(1)}(z)\right)=\mathscr{D}\left(K_{0}^{(1)}\right)$. It is enough to show that, for any $\varphi \in$ $\mathscr{D}\left(K_{0}^{(1)}\right)$ the mapping $\mathbb{C} \ni z \mapsto H^{(1)}(z) \varphi \in \mathscr{H}^{(1)}$ is analytic.

By the assumption (A5) we have, for any $(r, \theta) \in \mathbb{R}^{+} \times T$, that the power series $\tilde{v}_{z}(r, \theta)=\sum_{n \in \mathbb{N}_{0}} v^{(n)}(r, \theta) z^{n}$ with

$$
v^{(n)}(r, \theta)=\frac{1}{2 \pi \mathrm{i}} \oint_{|\zeta|=s} \frac{\tilde{v}_{\zeta}(r, \theta)}{\zeta^{n+1}} d \zeta
$$

for some $s>0$, has an infinite convergence radius. In addition, we clearly get from (66) that $\left|v^{(n)}(r, \theta)\right| \leq u_{2 s}(r, \theta) / s^{n}$ for any $(r, \theta) \in \mathbb{R}^{+} \times T$. In particular, we find that

$$
\left\|v^{(n)} \varphi\right\| \leq \frac{1}{s^{n}}\left\|u_{2 s} \varphi\right\|, \quad \varphi \in \mathscr{D}\left(K_{0}^{(1)}\right) .
$$

Therefore, for any $|z|<s$,

$$
v_{z} \varphi=\sum_{n \in \mathbb{N}_{0}} v^{(n)} z^{n} \varphi, \quad \varphi \in \mathscr{D}\left(K_{0}^{(1)}\right) .
$$

This concludes the proof since $s>0$ can be chosen arbitrarily large.

\section{References}

[1] M. Abramowitz and I. A. Stegun (eds.), Handbook of mathematical functions with formulas, graphs, and mathematical tables. U.S. Department of Commerce - U.S. Government Printing Office, Washington (DC), 1964. MR 0167642 Zbl 0171.38503

[2] J. Avron, I. Herbst, and B. Simon, Schrödinger operators with magnetic fields I. General interactions. Duke Math. J. 45 (1978), 847-883. MR 518109 Zbl 0399.35029

[3] A. Besch, Eigenvalues in spectral gaps of the two-dimensional Pauli operator. J. Math. Phys. 41 (2000), 7918-7931. MR 1796817 Zbl 0973.81020

[4] A. H. Castro Neto, F. Guinea, N. M. R. Peres, K. S. Novoselov, and A. K. Geim, The electronic properties of graphene. Rev. of mod. phys. 81 (2009), 109-162.

[5] P. R. Chernoff, Schrödinger and Dirac operators with singular potentials and hyperbolic equations. Pacific J. Math. 72 (1977), 361-382. MR 0510049 Zbl 0366.35031

[6] H. D. Cornean and G. Nenciu, On eigenfunction decay for two-dimensional magnetic Schrödinger operators. Comm. Math. Phys. 192 (1998), 671-685. MR 1620539 Zbl 0915.35013

[7] A. De Martino, L. Dell'Anna, and R. Egger, Magnetic confinement of massless Dirac fermions in graphene. Phys. Rev. Lett. 98 (2007), 66802. 
[8] A. De Martino and R. Egger, On the spectrum of a magnetic quantum dot in graphene. Semiconductor Science and Technology 25 (2010), 034006.

[9] R. Egger, A. De Martino, H. Siedentop, and E. Stockmeyer, Multiparticle equations for interacting Dirac fermions in magnetically confined graphene quantum dots. J. of Phys. A: Mathematical and Theoretical 43 (2010), 215202.

[10] L. Erdős, Gaussian decay of the magnetic eigenfunctions. Geom. Funct. Anal. 6 (1996), 231-248. MR 1384611 Zbl 0863.35089

[11] R. L. Frank, E. H. Lieb, and R. Seiringer, Stability of relativistic matter with magnetic fields for nuclear charges up to the critical value. Comm. Math. Phys. 275 (2007), 479-489. MR 2335782 Zbl 1135.81030

[12] D. Gilbarg and N. S. Trudinger, Elliptic partial differential equations of second order (second ed.). Grundlehren der Mathematischen Wissenschaften 224. Springer Verlag, Berlin etc., 1983. MR $0737190 \mathrm{Zbl} 0562.35001$

[13] W. Häusler and R. Egger, Artificial atoms in interacting graphene quantum dots. Phys. Rev. B 80 (2009), 161402.

[14] A. Iwatsuka, The essential spectrum of two-dimensional Schrödinger operators with perturbed constant magnetic fields. J. Math. Kyoto Univ. 23 (1983), 475-480. MR 0721381 Zbl 0564.35021

[15] H. Kalf, T. Ōkaji, and O. Yamada, Absence of eigenvalues of Dirac operators with potentials diverging at infinity. Math. Nachr. 259, 19-41. MR 2009334 Zbl 1038.35044

[16] A. Kormányos, P. Rakyta, L. Oroszlány, and J. Cserti, Bound states in inhomogeneous magnetic field in graphene: Semiclassical approach. Phys. Rev. B 78 (2008), 045430.

[17] H. Leinfelder, Gauge invariance of Schrödinger operators and related spectral properties. J. Operator Theory 9 (1983), 163-179. MR $0695945 \mathrm{Zbl} 0528.35024$

[18] E. H. Lieb and M. Loss, Analysis (second ed.). Amer. Math. Soc., Providence (RI), 2001. MR 1817225 MR 0966.26002

[19] S. Nakamura, Gaussian decay estimates for the eigenfunctions of magnetic Schrödinger operators. Comm. Partial Differential Equations 21 (1996), 993-1006. MR 1391530 Zbl 0855.35093

[20] E. Nelson, Analytic vectors. Ann. of Math. (2) 70 (1959), 572-615. MR 0107176 Zbl 0091.10704

[21] K. S. Novoselov, A. K. Geim, S. V. Morozov, D. Jiang, Y. Zhang, S. V. Dubonos, I. V. Grigorieva, and A. A. Firsov, Electric field effect in atomically thin carbon films. Science 306, 666-669.

[22] M. Ramezani Masir, P. Vasilopoulos, and F. M. Peeters, Graphene in inhomogeneous magnetic fields: bound, quasi-bound and scattering states. J. Phys.: Condensed Matter $\mathbf{2 3}$ (2011), 315301.

[23] M. Reed and B. Simon, Methods of modern mathematical physics IV. Analysis of operators. Academic Press, New York etc., 1978. MR 0493421 Zbl 0401.47001

[24] G. Rozenblum and N. Shirokov, Infiniteness of zero modes for the Pauli operator with singular magnetic field. J. Funct. Anal. 233 (2006), 135-172. MR 2204677 Zbl 1088.81046 
[25] G. Rozenblum and G. Tashchiyan, On the spectral properties of the Landau Hamiltonian perturbed by a moderately decaying magnetic field. In Ph. Briet et al. (eds.), Spectral and scattering theory for quantum magnetic systems. Proceedings of the conference, CIRM, Luminy, Marseilles, France, July 7-11, 2008. Amer. Math. Soc., Providence (RI), 2009, 169-186. MR 2655151 Zbl 1184.81063

[26] B. Simon, Quantum mechanics for Hamiltonians defined as quadratic forms. Princeton University Press, Princeton (NJ), 1971. MR 0455975 Zbl 0232.47053

[27] B. Simon, Maximal and minimal Schrödinger forms. J. Operator Theory 1 (1979), 37-47. MR 0526289 Zbl 0446.35035

[28] B. Simon, Trace ideals and their applications (second ed.). Amer. Math. Soc., Providence (RI), 2005. Zbl 1074.47001 MR 2154153

[29] V. Sordoni, Gaussian decay for the eigenfunctions of a Schrödinger operator with magnetic field constant at infinity. Comm. Partial Differ. Equations 23 (1998), 223-242. MR 1608520 Zbl 0898.34077

[30] B. Thaller, The Dirac equation. Texts and Monographs in Physics. Springer Verlag, Berlin etc., 1992. MR 1219537 Zbl 0765.47023

[31] V. Vogelsang, Absence of embedded eigenvalues of the Dirac equation for long range potentials. Analysis 7 (1987), 259-274. MR 928641 Zbl 0643.35073

[32] D. Wang and G. Jin, Magnetically confined states of Dirac electrons in a graphene-based quantum annulus, Europhysics Letters 88 (2009), 17011.

Received November 7, 2011

Martin Könenberg, Fakultät für Physik, Universität Wien, Boltzmanngasse 5, 1090 Vienna, Austria.

Previous address: Fakultät für Mathematik und Informatik, FernUniversität Hagen, Lützowstraße 125, D-58084 Hagen, Germany.

E-mail: martin.koenenberg@univie.ac.at

Edgardo Stockmeyer, Mathematisches Institut, Ludwig-Maximilians-Universität, Theresienstraße 39, D-80333 München, Germany.

E-mail: stock@math.lmu.de 